\title{
Bioinspired and biomimetic membranes for water purification and chemical separation: A review
}

\author{
Elham Abaie, Limeimei Xu, Yue-xiao Shen ( $ه)$
}

Department of Construction, Civil and Environmental Engineering, Texas Tech University, Lubbock, TX 79409, USA

\section{H I G H L I G H T S}

- The history of biological and artificial water channels is reviewed.

- A comprehensive channel characterization platform is introduced.

- Rationale designs and fabrications of biomimetic membranes are summarized.

- The advantages, limitations, and challenges of biomimetic membranes are discussed.

- The prospect and scalable solutions of biomimetic membranes are discussed.

\section{A R T I C L E I N F O}

\section{Article history:}

Received 29 August 2020

Revised 7 January 2021

Accepted 18 January 2021

Available online 10 March 2021

Keywords:

Aquaporins

Artificial water channels

Biomimetic membranes

Chemical separation and water purification

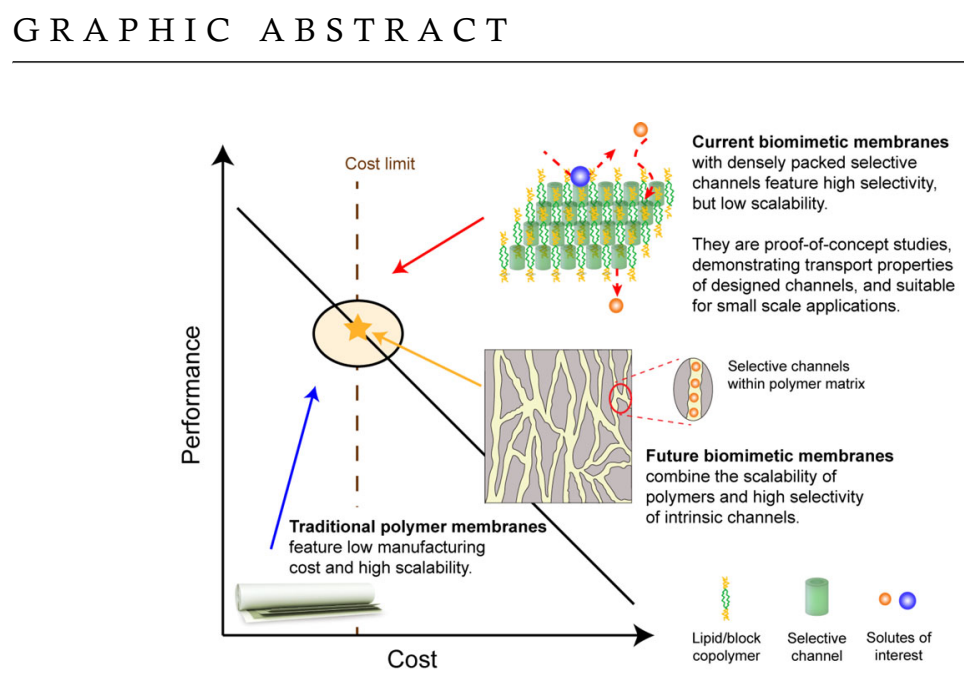

A B S T R A C T

Bioinspired and biomimetic membranes that contain biological transport channels or attain their structural designs from biological systems have been through a remarkable development over the last two decades. They take advantage of the exceptional transport properties of those channels, thus possess both high permeability and selectivity, and have emerged as a promising solution to existing membranes. Since the discovery of biological water channel proteins aquaporins (AQPs), extensive efforts have been made to utilize them to make separation membranes-AQP-based membranes, which have been commercialized. The exploration of AQPs' unique structures and transport properties has resulted in the evolution of biomimetic separation materials from protein-based to artificial channelbased membranes. However, large-scale, defect-free biomimetic membranes are not available yet. This paper reviews the state-of-the-art biomimetic membranes and summarizes the latest research progress, platform, and methodology. Then it critically discusses the potential routes of this emerging area toward scalable applications. We conclude that an appropriate combination of bioinspired concepts and molecular engineering with mature polymer industry may lead to scalable polymeric membranes with intrinsic selective channels, which will gain the merit of both desired selectivity and scalability.

C The Author(s) 2021. This article is published with open access at link.springer.com and journal.hep. com.cn

\section{Contents}

1 Introduction

2 Biological water channel proteins and artificial water channels
2.1 Biological water channel proteins and purification

Corresponding author

E-mail: yuexiao.shen@ttu.edu

\subsection{Artificial water channels}

3 Characterizations of biological and artificial water channels

3.1 Reconstitution of biological and artificial water channels and molecular transport studies

3.2 Molecular dynamics (MD) simulations of biological and artificial water channels in vesicle systems

4 Biological and artificial water channel-based membranes 
4.1 Vesicle-based membranes

4.1.1 Direct vesicle fusion

4.1.2 Charge-enhanced vesicle deposition

4.1.3 Chemical cross-linking

4.1.4 Interfacial polymerization

4.2 2-dimensional nanosheet-based membranes

4.2.1 2-dimensional nanosheet synthesis

4.2.2 Membrane fabrication

4.3 Lamellar block copolymer channel-based membranes

5 Engineered biological and artificial water channels

5.1 Pore engineering of biological water channels

5.2 Pore engineering of artificial water channels

6 Challenges and outlook

6.1 Scalability and practicability of current biomimetic membranes

6.2 Future direction of bioinspired and biomimetic membranes

\section{Introduction}

Since the discovery of biological water channels (BWCs) aquaporins (AQPs) (Preston et al., 1992; Chrispeels and Agre, 1994; Connolly et al., 1998; Agre, 2004) and the first study employing these biological molecules for desalination (Kumar et al., 2007), research on bioinspired and biomimetic membranes for water purification has flourished over the last two decades. Bioinspired and biomimetic membranes have been a long-lasting topic in membrane separation field and active in membrane conferences such as North American Membrane Society Annual Conference and International Congress on Membranes \& Membrane Processes. For water purification, the driving force is the global water and energy crisis and a critical need for energy-efficient water purification technologies (Shannon et al., 2008; Elimelech and Phillip, 2011; Werber et al., 2016b; Park et al., 2017; Epsztein et al., 2020). In addition to water purification, chemical separations are always challenging for energy and environmental applications (Gin, 2011; Sanders et al., 2013; Sholl and Lively, 2016; Koros and Zhang, 2017). Inspired by highly efficient biological transport systems, scientists and engineers breathe new life to the traditional membrane separation field: more energy-efficient and chemically versatile separations.

Over the past decade, AQP-based desalination membranes have been extensively tested in laboratory-scale studies (Wang et al., 2012; Zhao et al., 2012b; Tang et al., 2015). Aquaporin $\mathrm{A} / \mathrm{S}$ has commercialized this type of membranes (Aquaporin A/S; Tang et al., 2014; Jörg et al., 2015; Spulber and Gerstandt, 2018) (Fig. 1a). Using biological molecules to make biomimetic-hybrid membranes (Shen et al., 2014), this is the first stage and a milestone for bioinspired and biomimetic membranes.
After AQPs' structures and their water transport mechanisms were discovered, a myriad of artificial water channels (AWCs) have sprung up by chemists (Fig. 1b), which promotes the second epoch of biomimetic membranes: biomimetic-synthetic membranes (Shen et al., 2014). AWCs are aimed to mimic the structure and functionality of highly efficient BWCs and they have been vastly studied and reviewed recently (Barboiu and Gilles, 2013; Barboiu, 2016; Huo and Zeng, 2016; Kocsis et al., 2018b; Song et al., 2018; Song and Kumar, 2019). Research on AQPs and BWCs also brings methodologies from biophysics and related molecular transport concepts into the traditional membrane filtration field. Several high-performance biomimetic-synthetic membranes have been reported with remarkable permeability and selectivity (Shen et al., 2018; Lang et al., 2019; Tu et al., 2020) (Fig. 1b). These studies have further confirmed nature-inspired pore engineering at the molecular scale can result in the breakthrough of the permeability-selectivity trade-off of conventional polymeric membranes (Park et al., 2017). Now biomimetic membranes have come to a crossroad like other newly invented materials: how to find versatile applications and how to address scalability issues (Fig. 1c).

This tutorial review provides a comprehensive summary of bioinspired and biomimetic membranes developed over the past few years. It features a multidisciplinary methodology combined with biology, chemistry, material science and chemical engineering. The contents include preparation of building blocks for biomimetic membranes (e.g., protein purification and channel synthesis), design principles of bioinspired channels (e.g., pore engineering), a molecular transport characterization platform adopted and optimized from biophysics (experimental and simulation methods), membrane fabrication techniques specially tailored for biomimetic membranes, future outlook and scalable solutions of this nascent area. There have been several excellent reviews that have addressed various aspects of this field. Reviews by Tang (Tang et al., 2013), Hilal (Giwa et al., 2017) and Jeon (Fuwad et al., 2019) groups focused on AQP-based membranes for desalination. Nielsen's review summarized various applications of membrane proteins in lipid and block copolymer and membranes (Hélix-Nielsen, 2018). Jiang's article covered all aspects of bioinspired membranes, not limited to separation (Zhao et al., 2014b). Escobar's recent work introduced different biological and artificial pores as the building blocks and their potential applications for biomimetic membranes (Wagh and Escobar, 2019). Kumar's recent review more emphasized on optimized fabrications of biomimetic membranes (Song et al., 2019). Elimelech group opted a quantitative method in their recent publication, to assess biomimetic membrane formats (Porter et al., 2020). Despite the listed reviews focused on different topics, an end-to-end, comprehensive overview of biomimetic membranes does not exist. This review is a continuation of the first comprehensive biomimetic 
(a)

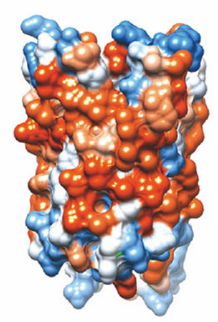

Aquaporin 1

Polyamide layer AQP-embedded vesicle

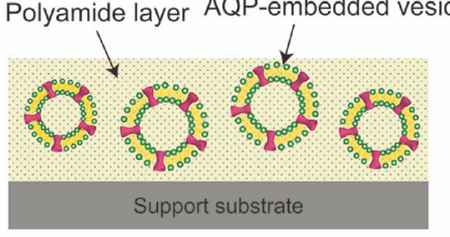

AQP-based desalination membrane

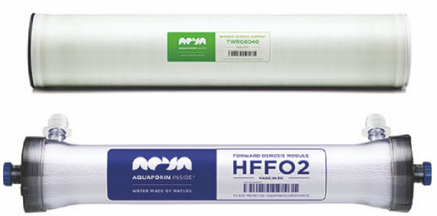

Aquaporin Inside ${ }^{\circledR}$ membrane by Aquaporin A/S (b)

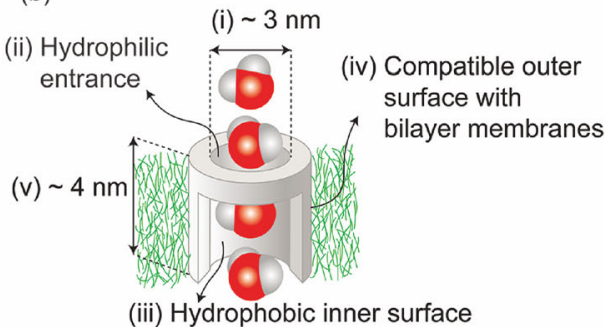

Artificial water channels

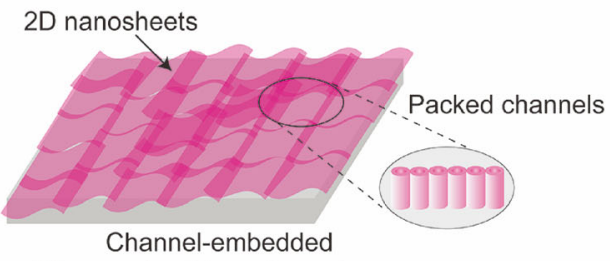

2D nanosheet-based membrane

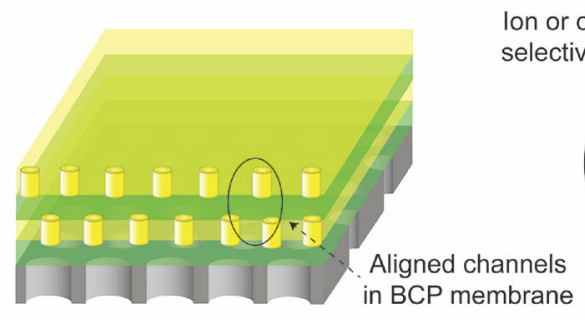

Lamellar BCP channel-based membrane (c)

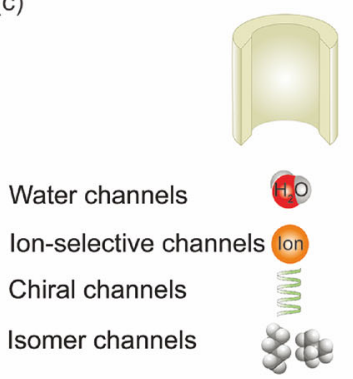

Future channel design
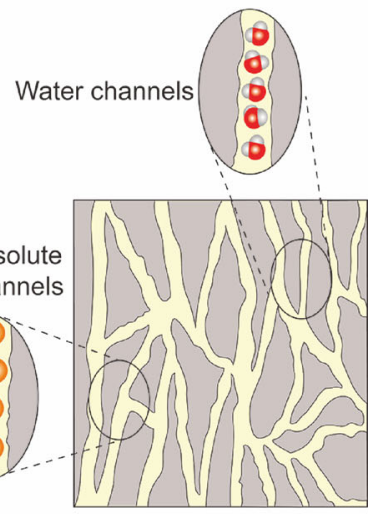

Scalable polymer membranes with intrinsic selective channels

Fig. 1 Three stages of bioinspired and biomimetic membranes for water purification and chemical separation. (a) Aquaporin (AQP)based membranes for desalination applications. AQPs show excellent water permeability and selectivity. AQP-incorporated vesicles are embedded in the polyamide selective layer of traditional thin film composite (TFC) membranes. The resultant membranes are the firstgeneration biomimetic membranes and commercialized by Aquaporin A/S. Reproduced with permission from Aquaporin A/S. (b) Channel-based membranes for angstrom-scale separations. Studies on AQPs have promoted the research on artificial structures (i.e., artificial water channels, AWCs) that have AQP-like structure, permeability, and selectivity. These channels have been made into 2dimensional (2D) arrays with high packing density and the 2D nanosheet-based TFC membranes have outperformed comparable commercial membranes. Another strategy is to use the compatibility between AWCs and amphiphilic block copolymers (BCPs) to make lamellar channel-based membranes which are solvent casting-based and more scalable. (c) Highly selective channels and more scalable polymeric membranes with intrinsic channel-like structures. Innovative channels are expected to target specific niches in separation fields. Practically, these structures should shed light on the designs of traditional polymer membranes to improve separation efficiency, while maintaining their own scalability.

membrane review work (Shen et al., 2014) and provides such a summary covering all aspects for biomimetic membranes. In additional to the technical part, we provide a unique insight into the scalability challenges. The latter is particularly critical when a technology has been invented for years but practicability may become an unbridgeable gap between the novel materials and scalable applications.

\section{Biological water channel proteins and artificial water channels}

BWCs are the central building blocks of biological cell membranes to control water transport. How to mimic such structures is a key step for making bioinspired and biomimetic membranes. Historically, these biological units were first discovered and well characterized, and directly used for fabricating prototype biomimetic membranes. Their structures were later mimicked in AWCs, which improved upon them through relatively simple synthesis and chemical stability.

\subsection{Biological water channel proteins and purification}

The discovery of AQPs in living organisms shows significant importance to biophysics and human health (Agre et al., 2002). They have become model molecules for scientists and engineers to study and mimic (Barboiu, 2016; Werber et al., 2016b; Song et al., 2018). AQPs are omnipresent in cell membranes of different living organisms such as bacteria, fungi, animals, and plants (Gomes 
et al., 2009). They are responsible for balancing osmotic pressure across cell membranes. Most of the proteins in this family only transport water molecules while rejecting other solutes. AQPs consist of six $\alpha$-helix domains that form a membrane-spanning pore with multiple hydrophobic amino acid residuals lining within the lumen (Verkman and Mitra, 2000) (Fig. 2a). The beauty of their highly selective channel-mediated water transport can be ascribed by four main factors. First, the hourglass shape of AQPs by the six domains enables size exclusion at the narrowest region with an $\sim 3 \AA$ aperture which results in rejection of most hydrated ions and other solutes (Agre, 2004; Barboiu, 2016). Secondly, the positively charged Arg 185 near the selectivity site contributes to the electrostatic repulsion of cations (Murata et al., 2000). Moreover, the internal hydrophobic environment contributed by the multiple hydrophobic amino acid residuals along the inner wall facilitates rapid water conductance which acts as a similar mechanism 'slip flow' as found in carbon nanotubes (CNTs) (Kruse et al., 2006; Kumar et al., 2013; Tunuguntla et al., 2017; Song et al., 2018). Finally, water dipole rearrangement aided by several amino acid groups (Asn76 and Asn192 in two Asn-Pro-Ala motifs in the middle of the protein) blocks the formation of a continuous water wire along the pore and prevents proton transport, thus making AQPs strict water channels (Agre, 2004). Other membrane proteins (MPs) such as outer membrane protein F (OmpF) (Hancock and Carey, 1979), ferric hydroxamate uptake protein component A (FhuA)
$\Delta \mathrm{C} / \Delta 4 \mathrm{~L}$ (Mohammad et al., 2011), $\alpha$-hemolysin ( $\alpha \mathrm{HL}$ ) (Branton et al., 2008) have been used to study biomimetic membranes. They are $\beta$-barrel proteins with pore sizes from $0.8 \mathrm{~nm}, 1.3 \mathrm{~nm}$, and $1.5 \mathrm{~nm}$, respectively (Figs. 2b-2d). OmpF is a special class of outer membrane proteins that form water-filled channels and can transport molecules with molecular weight (MW) less than $600 \mathrm{Da}$ (Masi and Pagès, 2013). FhuA $\Delta \mathrm{C} / \Delta 4 \mathrm{~L}$ is an engineered protein which results from removing almost one third of the total amino acids of wild type FhuA protein. As a result, the entire cork domain and four extracellular loops are encompassed to form a rigid transmembrane pore (Mohammad et al., 2011). aHL from Staphylococcus aureus is a self-assembled toxin that binds to the plasma membrane of a susceptible cell. It oligomerizes and forms a water-filled transmembrane channel, and may cause death to the host due to the uncontrolled permeation of water, ions, and small organic molecules (Aksimentiev and Schulten, 2005). Because of the relatively large pore sizes, these three proteins are capable of transporting small solutes across cell membranes and cannot be considered as strict BWCs.

Compared to the tortuous, discontinuous channels within the polyamide network of commercial reverse osmosis (RO) membranes (Kumar et al., 2013), over billions of years' evolution in biology bestows BWCs with delicate structures that possess exceptional water permeability and selectivity. For scientists and engineers, AQPs' structures have shed light on bioinspired energy-efficient

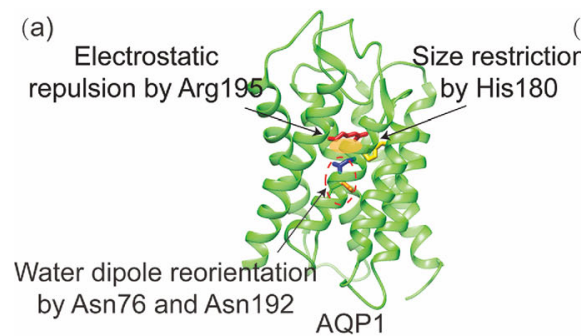

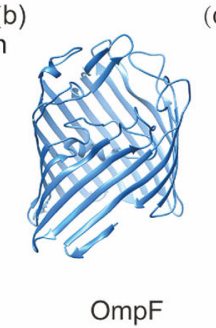

(c)

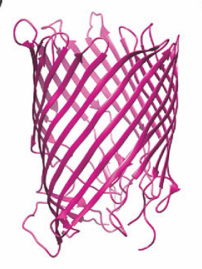

FhuA $\Delta \mathrm{C} / \Delta 4 \mathrm{~L}$

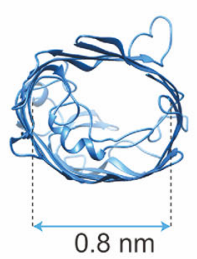

(d)
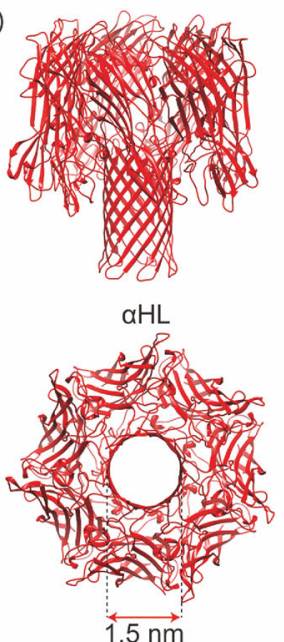

Fig. 2 Several representative biological water channels used for biomimetic membranes. (a) Aquaporin 1 (AQP1) is one representative protein of aquaporins (AQPs) family. Several key pore-lining amino acid residuals are responsible for its high water permeability and selectivity. Within the pore, $\operatorname{Arg} 195$ and His 180 form the narrowest part of the channel $(\sim 3 \AA)$, slightly higher than the diameter of a water molecule $(2.7 \AA)$. Arg195 is also responsible for electrostatic repulsion. Asn76 and Asn192 control the water dipole rearrangement in two Asn-Pro-Ala motifs near the size exclusion region, which blocks proton transport. (b) Outer membrane protein $\mathrm{F}(\mathrm{OmpF})$ is a $\beta$-barrel membrane protein that has a pore size of $0.8 \mathrm{~nm}$. (c) Ferric hydroxamate uptake protein component A $\Delta \mathrm{C} / \Delta 4 \mathrm{~L}$ (FhuA $\Delta \mathrm{C} / \Delta 4 \mathrm{~L}, \Delta \mathrm{C} / \Delta 4 \mathrm{~L}$ indicates the deletion of the cork domain and four large extracellular loops) is a stiff engineered biological transmembrane nanopore with a pore size of $1.3 \mathrm{~nm}$. (d) $\alpha$-Hemolysin ( $\alpha \mathrm{HL}$ ) is a water-soluble $33 \mathrm{kDa}$ monomer secreted by Staphylococcus aureus. It assembles into a heptamer to form a transmembrane pore of $1.5 \mathrm{~nm}$ on a target membrane. 
desalination filtration materials (Kumar et al., 2007; Wang et al., 2012; Zhao et al., 2012b; Tang et al., 2013; Werber et al., 2016b; Song and Kumar, 2019). Molecular design of desalination membranes from biomimetic perspective is formidable due to limited available building blocks as well as from synthesis itself. Therefore, the first step is directly using biological components AQPs as the building blocks to fabricate membranes as a proof of concept (Zhao et al., 2012b). Compared to traditional polymeric membrane synthesis, utilizing biological component imposes tremendous difficulties. For biophysics studies, these AQPs can be usually extracted in small quantities from native tissues or bacteria cells (Agre, 2004) (e.g., Aquaporin 1 (AQP1) from kidney (Sabolic et al., 1992), Aquaporin 2 from collecting ducts (Nephrol, 1998), Aquaporin 0 (AQP0) from eye lenses (Virkki et al., 2001), and Aquaporin Z (AqpZ) from bacteria cells (Calamita et al., 1995)) and characterized in vitro. For engineering applications, large quantities of proteins are needed for subsequent characterizations and fabrications (Wagner et al., 2006). This requires genetic and chemical engineering of protein expression and downstream purification process. The first procedure is to transfer the plasmids encoded with AQPs of interest into engineered E. coli (Borgnia et al., 1999a), other bacteria such as Rhodobacter sphaeroides (Erbakan et al., 2014) or yeast (Saboe et al., 2017) for overexpression. AQPs, either from native tissues or overexpressed engineered cells, are extracted together with membrane fractions using common cell lysis procedures such as sonication, French press, and microfluidizer (Fig. 3). The proteins embedded within the membrane fractions from the crude extract are separated from cell debris and soluble parts using consecutive centrifugations. The membrane fractions, pelleted from ultracentrifugation because of the similar density to water, are solubilized with detergent. AQPs are then stabilized in detergent and purified through chromatography based on size, charge, or specific binding (Scopes, 1982; Ersson et al., 2011). A common technique is to attach 6 histidine residues to the $\mathrm{N}$-terminal of AQPs and the modified His-tagged AQPs can be efficiently enriched with high purity by nickel or cobalt-based resins (Bornhorst and Falke, 2010). Other MPs such as OmpF and FhuA $\Delta \mathrm{C} / \Delta 4 \mathrm{~L}$ can be expressed, extracted and purified in this approach as well (Hancock and Carey, 1979; Plançon et al., 1997). Because these proteins are MPs, the expression level is relatively low. Additionally, poor stability and high cost of using specific detergents during the purification impede large-scale commercialized production of MPs for engineering applications (Hovijitra et al., 2009; Li et al., 2012; Shen et al., 2014; Giwa et al., 2017). To date, most of AQPbased biomimetic membranes use AqpZ expressed from E. coli and a commercial company Aquaporin A/S, Denmark, fabricates AqpZ-functionalized RO and forward osmosis (FO) membranes (Fig. 1c) (Aquaporin A/S). Other MPs are mostly expressed in laboratories and used to make
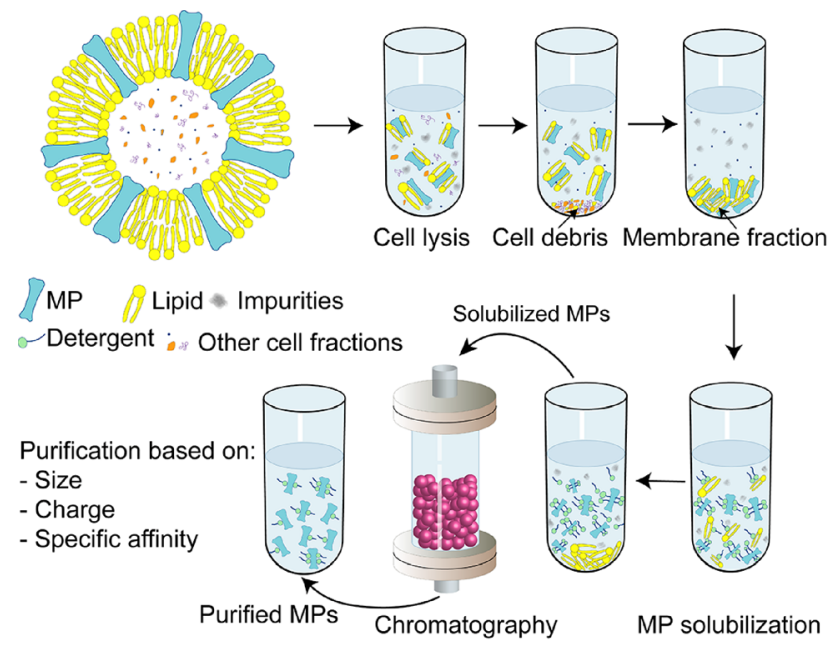

Fig. 3 A schematic illustration of membrane protein (MP) expression and purification from E. coli. Cultivated and overexpressed E. coli is subject to cell lysis to break down cells and extract MPs with other membrane fractions. Several consecutive centrifugations will be conducted to separate MPs and membrane fractions from cell debris and solute parts. After stabilization of MPs in detergent, they will be separated from lipids and further purified through chromatography based on size, charge, or specific binding.

biomimetic membranes for other applications such as sensoring (Hélix-Nielsen, 2009).

\subsection{Artificial water channels}

Despite high water permeability and selectivity of BWCs, the structural instability and low processability from the proteins themselves hinder their broad engineering applications at larger scales. Studies on AQP-based membranes are a proof of concept using biological materials as building blocks to make separation membranes that possess certain properties of highly permeable and selective biological membranes (e.g., biomimetic-hybrid membranes) (Kumar et al., 2007; Wang et al., 2012; Zhao et al., 2012b; Shen et al., 2014). The ultimate goal is to replace these structures in a synthetic manner. The transition step from BWCs to synthetic structures results in the birth of AWCs (Barboiu, 2016; Song et al., 2018; Song and Kumar, 2019). Studies on AWCs can be traced back to the early 2000s, after AQPs' structures were discovered. One line stems from synthetic ion channels (Sisson et al., 2006; Fyles, 2007; Matile et al., 2011; Sakai and Matile, 2013) and is focused on the structures that are capable of translocating water molecules. The other line is CNTs. CNTs have been regarded as potential water filtration materials due to extreme fast water conductance (Hinds et al., 2004), but the linkage with AWCs was generally accepted when carbon nanotube porins (CNTPs) were introduced (Geng et al., 2014; Tunuguntla et al., 2016b, 2016a, 2017; Sianipar et al., 2017; Sanborn et al., 
2018; Chen et al., 2019; Yao et al., 2019; Sullivan et al., 2020). These special CNTs have subnanometer pores with a columnar length of a few nanometers that can be inserted into lipid bilayer membranes. In this regard, AWCs, including CNTPs, are synthetic structures with subnanometer pores and similar dimensions as biological MPs. Current channels can be classified into self-assembled and unimolecular channels. They mimic (or partially mimic) the structural features of AQPs and can be incorporated into 3-4 nm thick lipid or block copolymer (BCP) membranes with vertical (or close to vertical) orientations (Fig. 1b). Water permeability and selectivity of AWCs have gradually approached AQPs' performances over the last decade's research. However, current AWCs are still not as perfect as AQPs because of the difficulties in accurately replicating the key selectivity site of AQPs, available structures of subnanometer pores as well as synthesis challenges. There are a few excellent reviews on this topic (Barboiu, 2012, 2016; Huo and Zeng, 2016; Werber et al., 2016a, 2016b; Song et al., 2018, 2019; Song and Kumar, 2019; Epsztein et al., 2020). For this review, we will cover how AWCs evolved over the past few years, and how to make biomimetic membranes around AWCs. There are two main challenges of current AWC studies: 1) fundamental design and synthesis, and 2) practical implementation into filtration materials. In this section, we will first review several AWC structures, and discuss the design and synthesis challenges. The characterization and membrane fabrication will be included in Sections 3 and 4. A chronological overview of the synthesized channels so far and their key structures are displayed in Fig. 4. Their performances are summarized in Table 1.

The first AWC is the zwitterionic coordination polymer synthesized by the reaction of carboxyl-functionalized imidazolium salt N,N'-diacetic acid imidazolium bromide with zinc, published by Dyson and Antonijevic groups in 2005 (Fig. 4a) (Fei et al., 2005). The polymer formed a helical tubular structure with an $\sim 2.6 \AA$ pore, as revealed by $\mathrm{X}$-ray diffraction and solid-state nuclear magnetic resonance measurements. The helical structure was formed by bridging the dicarboxylate anions linked to the zinc polymer units, and further supported by the weak $\pi-\pi$ stacking interactions between the imidazolium moieties and intrahelical hydrogen bonds. A full cycle within the helix was composed of the two polymer molecules with an intralayer distance of $6.2 \AA$. The channel accommodated a $\begin{array}{ll}\text { (a) Zwitterionic polymers } & \text { (b) Dendritic dipeptides }\end{array}$

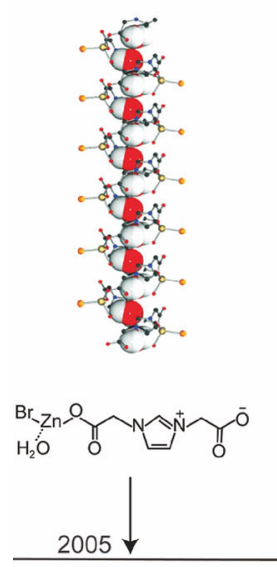

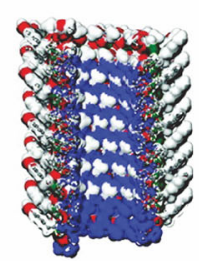

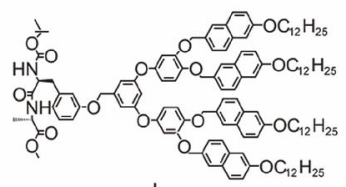

(d) Pillar[5]arene channel

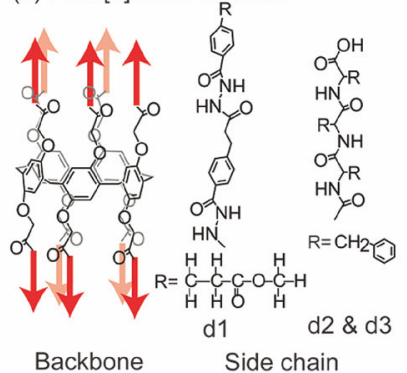

d1: Hydrazide-appended pillar[5]arenes d2: Peptide-appended pillar[5]arenes d3: Peptide-appended pillar[5]arenes ( $p R$ and $p S$ ) (f) Macrocycle-stacked channel (g)

(g) Hybrid[4]arene channel

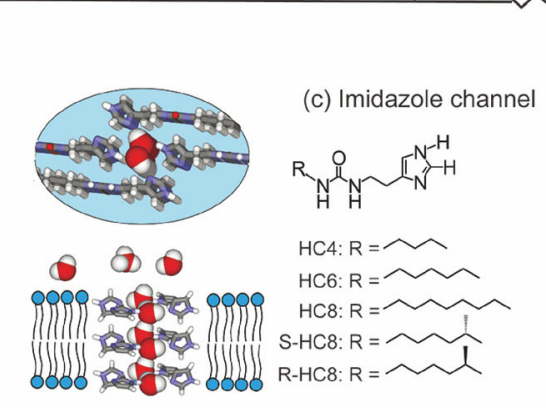

\section{.}

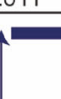

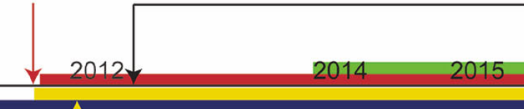

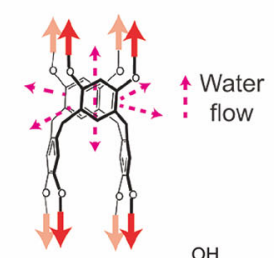

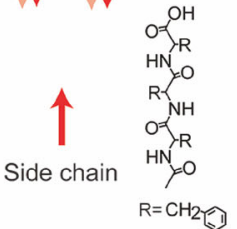

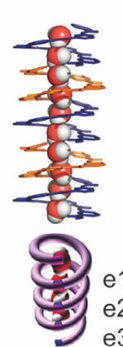

(e) Aquafoldmer channel
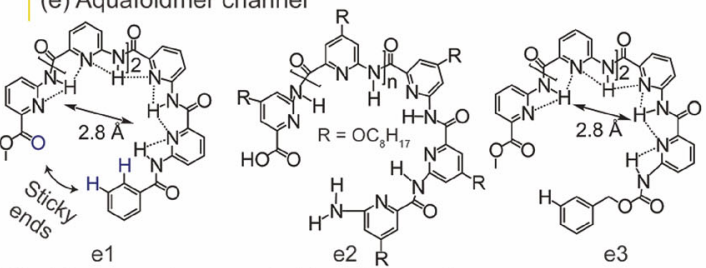

(h) Carbon nanotube porins

e1: Pyridine-based, connected by sticky ends

e2: Polypyridine-based, connected by covalent bonds

e3: Pyridine-based, pore volume expanded by $20 \%$ vs. e1, connected by sticky ends

Fig. 4 Artificial water channels developed over the last two decades. (a) Helical tube formed by zwitterionic coordination polymers. Reproduced with permission from Fei et al., 2005. Copyright 2005 John Wiley and Sons. (b) Helical pore assembled by dendritic dipeptides. Reproduced with permission from Kaucher et al., 2007. Copyright 2007 American Chemical Society. (c) Imidazole I-quartets water channels. Reproduced with permission from Barboiu, 2016. Copyright 2016 Royal Society of Chemistry. (d) Pillar[5]arene-based water channels. (e) Aquafoldamer-based water channels. Reproduced with permission from Zhao et al., 2014. Copyright 2014 American Chemical Society. (f) $m$-Phenylene ethynylene macrocycle-stacked channels. (g) Peptide-appended hybrid[4]arene water channels. (h) Carbon nanotube porins. Image credit by F. Aydin, A. Pham, and A. Noy from Lawrence Livermore National Laboratory. 
Table 1 Pore size, water permeability and salt rejection of artificial water channels

\begin{tabular}{|c|c|c|c|c|}
\hline Artificial water channels & $\begin{array}{l}\text { Pore size } \\
(\AA)\end{array}$ & $\begin{array}{c}\text { Water permeability } \\
\left(\mathrm{H}_{2} \mathrm{O} \mathrm{s}^{-1} \text { per channel }\right)\end{array}$ & $\begin{array}{c}\text { Salt } \\
\text { rejection }\end{array}$ & References \\
\hline Zwitterionic polymers & 2.6 & NA & NA & Fei at al., 2005 \\
\hline Dendritic dipeptides & 14.5 & NA & $\times$ & Kaucher at al., 2007 \\
\hline Imidazole channels & 2.6 & $1.5 \times 10^{6}$ & $\sqrt{ }$ & $\begin{array}{l}\text { Leduc et al., 2011; } \\
\text { Licsandru et al., 2016; } \\
\text { Schneider et al., } 2017\end{array}$ \\
\hline \multicolumn{5}{|l|}{ Pillar[5]arene channels* } \\
\hline Hydrazide-appended (d1) & 4.7 & $\sim 40$ & $x$ & Hu et al., 2012 \\
\hline Peptide-appended (d2) & 4.7 & $3.5 \times 10^{8}$ & $\times$ & $\begin{array}{l}\text { Chen et al., 2013; } \\
\text { Shen et al., } 2015\end{array}$ \\
\hline Peptide-appended (pR) (d3) & 4.7 & $1.3 \times 10^{9}$ & $\sqrt{ }$ & Li et al., 2019 \\
\hline \multicolumn{5}{|l|}{ Aquafoldmer channels* } \\
\hline Pyridine-based (e1) & 2.8 & $2.2 \times 10^{8}$ & $\sqrt{ }$ & Zhao et al., 2014 \\
\hline Polypyridine-based (e2) & 2.8 & $1.6 \times 10^{9}$ & $\sqrt{ }$ & Shen et al., 2020a \\
\hline Pore volume optimized (e3) & 2.8 & $3 \times 10^{9}$ & $\sqrt{ }$ & Shen et al., 2020b \\
\hline$m$-phenylene ethynylene macrocycle-stacked channels & 6.4 & $5 \times 10^{7}$ & $\times$ & Zhou et al., 2012 \\
\hline Peptide-appended hybrid[4]arene PAH[4] & $\sim 3$ & $>10^{9}$ & $\sqrt{ }$ & Song at al., 2020 \\
\hline \multicolumn{5}{|l|}{ Carbon nanotube porins } \\
\hline Narrow & 8 & $2.8 \times 10^{10}$ & $\times$ & Tunuguntla et al., 2017 \\
\hline Wide & 15 & $2 \times 10^{9}$ & $\times$ & Tunuguntla et al., 2017 \\
\hline
\end{tabular}

*The corresponding structures of $\mathrm{d} 1-\mathrm{d} 3$ and e1-e 3 can be found Fig. 4.

single-file water chain inside. No water permeability test was conducted, but the authors hypothesized that the water transport rate should be much lower compared to natural water channels because of the presence of hydrogen bonds with the encapsulated water molecules. In 2007, Percec and coworkers published another helical water channel assembled by dendritic dipeptides with enhanced intermolecular peripheral $\pi-\pi$ stacking (Kaucher et al., 2007) (Fig. 4b). This study first incorporated the channel into a vesicle system for molecular transport characterizations, with the assistance of visual optical microscopy and fluorescent assay. Water and proton transports were identified but no quantitative data was measured. With a pore diameter of $14.5 \AA$, this channel was not expected to reject salt.

Following the pioneering work, significant progress has been made on the exploration of new AWC architectures, due to a deeper understanding of AQPs' structures, breakthrough in synthesis, more established characterization platforms, as well as the assistance from computational simulations. Imidazole I-quartet channels (Fig. 4c) by Barboiu group (Leduc et al., 2011; Licsandru et al., 2016; Schneider et al., 2017), pillar[5]arene channels (Fig. 4d) by Hou group (Hu et al., 2012; Chen et al., 2013; Shen et al., 2015, 2018; Li et al., 2019a), aquafoldamer channels by Zeng group (Fig. 4e) (Zhao et al., 2012a, 2014a; Huo and Zeng, 2016; Shen et al., 2020a, 2020b) and macrocyclic channels by Gong group (Fig. 4f) (Zhou et al., 2012) are the representative examples. The imidazole channels were made of imidazole quartets with urea ribbons stacking on top of each other by inner $\pi-\pi$ stacking and strong hydrophobic interactions (Leduc et al., 2011). They are the first channels that are capable of facilitating water permeation while achieving $\sim 100 \%$ salt rejection because the interior gap of the channel $(2.6 \AA)$ was very close to the diameters of some narrow AQPs (Licsandru et al., 2016). The water conductance was low due to the hydrogen bond interactions between water molecules and the inner surface of the I-quartets. They also discovered that chirality not only influenced the channel's stability in lipid membranes, but also preserved the total dipolar orientation and ordering of the encapsulated water wires (Licsandru et al., 2016; Schneider et al., 2017). Pillar [5]arene channels are the first unimolecular AWCs. The channels were constructed on the recently discovered macrocycle pillar[5]arene (Ogoshi et al., 2008, 2016; Cragg and Sharma, 2012). The macrocyclic ring has a pore diameter of $4.7 \AA$, and by anchoring different side chains, this macrocycle could be transformed into a tubular structure with 3-4 nm in length. The as-synthesized channel can be inserted into bilayer membranes just like biological transmembrane proteins. The first pillar[5]arene AWCs are hydrazide-appended pillar[5]arenes (Fig. 4d, d1) ( $\mathrm{Hu}$ et al., 2012). The hydrazide side chains were stabilized by intermolecular hydrogen bonds. Water transport was observed, but the water wire was disrupted 
due to the alternative hydrophobic/hydrophilic domains along the cylindrical channel structure. The extra hydrogen bonds also retarded water mobility. The second-generation pillar[5] arene channels are peptide-appended pillar[5] arenes (PAP[5]) (Fig. 4d, d2). By substituting with more hydrophobic phenylalanine-based peptides as side chains (Chen et al., 2013), the single-channel water permeability was significantly enhanced from original $\sim 40$ ( $\mathrm{Hu}$ et al., 2012 ) to $3.5 \times 10^{8}$ water molecules per second (Shen et al., 2015). The solute rejection showed this channel behaved like a pore of 500 Da molecular weight cut-off (MWCO) (Shen et al., 2015). Although not a perfect AWC, taking the advantage of its unimolecular form, Hou and Kumar groups packed PAP[5] channels into high-density 2dimennsional (2D) arrays (Shen et al., 2015), and synthesized the first AWC-based biomimetic membranes (Shen et al., 2018). Wang group later adopted the chirality idea and made another version of pillar[5]arene-based channels and improved the water permeability by $\sim 1$ order of magnitude (Fig. 4d, d3) (Li et al., 2019a). Aquafoldamer channels are a series of pyridine-based oligomers with chiral helical structures (Fig. 4e) (Zhao et al., 2014a; Shen et al., 2020a, 2020b). These channels are formed by either self-assembly of these oligomers with specially designed sticky ends (Zhao et al., 2014a; Shen et al., 2020a) (i.e., interaction between ester and benzyloxycarbonyl groups), or through polymerization (Shen et al., 2020b). The inner diameter of the channels is $\sim 2.8 \AA$, which is the similar size compared to the narrowest cavity of AQPs. The first channel by Zeng group had very low yield and did not show prominent water permeability under osmotic gradient, but the helical stacks were found to effectively transport water in the context of a proton gradient (Fig. 4e, e1) (Zhao et al., 2014a). By tuning the backbones, their polypyridine-based aquafoldamer was able to transport water at a rate of $1.6 \times 10^{9}$ water molecules per second (Fig. 4e, e2) (Shen et al., 2020a). Their latest aquafoldamer channel expanded the pore volume by $20 \%$ and achieved a permeability of $\sim 3 \times 10^{9}$ water molecules per second (Fig. 4e, e3) (Shen et al., 2020b). Gong group synthesized $\pi$-conjugated hexa $(m$-phenylene ethynylene) macrocyclic channels, with an inner diameter of $6.4 \AA$ (Fig. $3 \mathrm{f}$ ). One of these channels was reported to approach AQP1's water permeability (Fig. 4f) (Zhou et al., 2012).

The most recently published AWC structure is peptideappended hybrid[4]arene (PAH[4]) channel (Song et al., 2020). The synthesis was similar to pillar[5] arene-based channels (Chen et al., 2013; Shen et al., 2015) via attaching D-L-D phenylalanine tripeptides onto the macrocyclic ring (Fig. 4g). The difference was the smaller cavity of the hybrid[4]arene macrocycle $(\sim 3 \AA \times \sim 5 \AA$ ) (Fig. 4g) that favored both water permeation and salt exclusion. Another difference compared to traditional tubular AWCs was that this channel opened more lateral water flow pathways through outward void windows with dimensions of 5-7 $\AA$ around the channel (Fig. 4g, see water flow path), in addition to the postulated vertical water transport. Therefore, this PAH[4] should be regarded as a water channel cluster or network instead of a single channel when incorporated in bilayer membranes. The interconnection of these void windows with neighboring channels enhanced the overall water permeability to $\sim 4 \times 10^{9}$ water molecules per second while maintaining high salt rejection. This network structure should shed light on future polymeric membrane design at the molecular engineering level.

CNTs have been shown to possess efficient water transport properties (Hinds et al., 2004; Holt et al., 2006). They were processed into CNTPs by Noy group (Geng et al., 2014; Tunuguntla et al., 2017). CNTPs were fabricated by sonication-assisted cutting of purified long CNTs, making them into short porins with 6 to $10 \mathrm{~nm}$ in length (Fig. 4h). The CNTPs have been demonstrated high biocompatibility and structural stability and they behaved like other transmembrane channels. The hydrophilic groups on the tube rim interacted with hydrophilic lipid headgroups which made insertion of CNTPs into lipid bilayers favorable (Geng et al., 2014). Noy group compared two CNTPs (0.8 nm vs. $1.5 \mathrm{~nm}$ in diameter) (Tunuguntla et al., 2017). They found in the narrower CNTPs, water molecules were prone to be confined into single-file wires. The encapsulated water molecules had less hydrogen bond interactions, thus further lowering the energy barrier for water transport enhanced by the hydrophobic inner surface of the CNTPs. The water permeability of the narrow CNTPs was 1 order of magnitude higher than the wider version, and also achieved approximately 6-fold enhancement compared to AQP1 (Verkman and Mitra, 2000), resulting the first AWC that outperformed AQPs in terms of water permeability.

Current AWC designs follow a few simple guidelines based on AQPs' structures (Fig. 1b). First, an angstromsized pore is needed. $3 \sim 4 \AA$ at the narrowest site of the aperture is required for salt rejection. Imidazole channels (Leduc et al., 2011; Licsandru et al., 2016), aquafoldamer channels (Shen et al., 2020a; 2020b) and PAH[4] channels (Song et al., 2020) can achieve high salt rejection because of the appropriate size exclusion while the other candidates with larger pore sizes have been found to be less efficient in excluding salts. Secondly, the internal hydrophobic requirement within channels is necessary for fast water translocation. The number of hydrogen bonds formed between water molecules and adjacent hydrophilic motifs (especially oxygen and nitrogen atoms) should be limited (Horner et al., 2015). The earlier imidazole channels formed excess hydrogen bonds that retarded their water mobility (Leduc et al., 2011). The pillar[5]arene family showed that the water transport was much faster when the side chains were made of hydrophobic amino acid peptide (phenylalanine) (Shen et al., 2015) than hydrophilic hydrazide (Hu et al., 2012). To achieve faster water transport, the inner pore environment like CNTPs is ideal since water molecules can move freely without friction 
(Tunuguntla et al., 2017). Thirdly, the entrance of AWCs should be hydrophilic to reduce the energy barrier for water entry. Moreover, pore structure should be rigid enough to remain open to accommodate movable water molecules. PAP [5] side chains were floppy so that the pores were found to be blocked by its side chains during simulation, which reduced the water permeability (Shen et al., 2015). Finally, AWCs should have structural compatibility with lipid or BCP membranes (i.e., minimum physical and chemical hydrophobic mismatches, as discussed in Section 4.2.1). AWCs are supposed to have a columnar length of 3-5 $\mathrm{nm}$ and hydrophobic outer surface. The final guideline is specific to channel studies if the channels are designed to mimic biological channels and studied in bilayer systems (e.g., biological or BCP membranes). If we step back and look from the perspective of separation membranes, this rule may not be necessary. A more practical concern is how to transplant these highly selective structures into polymer membrane matrix, which will be discussed in later sections.

Despite these simple guidelines and several attempts to mimic BWCs, there are no universal rules for AWC design and synthesis. First, we lack the knowledge of available angstrom-scale pore structures, especially in the range of 3-4 A. Conversely, such structures can be easily templated by self-assembled protein subdomains. Current AWCs are limited to several types of structures. The subsequent synthesis is more difficult with relatively low yields. Take PAP [5] channels as an example, they require a 10-step synthesis with the yield of most steps around $80-90 \%$. One step regarding the cross-link between the pillar[5]arene ring and 10 peptide chains results in a low yield of $10 \%$ $20 \%$, making the whole synthesis yield less than $10 \%$. Secondly, the designs could be a matter of trial and error. The early studies often adopted one major design with several possible derivatives, and one of the designs finally worked experimentally. The post-simulation explained why such design was reasonable. Another challenge during design is the single-file water transport. The fast single-file water movement within AQPs is balanced by the limited hydrogen bonds and hydrophobic frictionless environment, which are contributed by a selected group of amino acid residuals with specific orders. Current AWC architectures can hardly hit that sweet spot yet. Combined with computational simulations, future channel design can get closer to AQPs. However, the subsequent synthesis difficulties still remain. Finally, we are also hindered by the available methods to incorporate these channels into polymer membrane matrix with appropriate orientation and maximum reconstitution density; thus the scalability is also low and problematic. One reason for such difficulties in making channel-based polymer membranes is probably the protein-like shapes of these AWCs. They are ideal for molecular transport studies in biological membranes but not optimized for material synthesis. With these configurations, they need to be aligned in a particular environ- ment. We have to follow self-assembly principles and these membranes are non-scalable so far.

In summary, the early channels (e.g., zwitterionic polymers, dendritic dipeptides and the first generation of pillar[5]arene channels) were just proof of concept studies showing these synthetic transmembrane pores with water wire encapsulated. The channels were then optimized to possess quite fast water conductance. The pore sizes of newer channels are within subnanometer ranges. When the pore size is close to $0.3 \mathrm{~nm}$, the resulting channels can effectively reject salt. They can be used for desalination. The channels with larger pore sizes can translocate salt and other solutes, which means they are not strictly water channels and can be used for other filtration applications such as nanofiltration (NF) and angstrom-scale separation. These characteristics are included in Table 1 and the later Sections (4.1 and 4.2) of the manuscript will discuss the efforts on incorporating these channels into membrane materials.

\section{Characterizations of biological and artificial water channels}

Compared to traditional membrane separation materials, the platform to determine the permeability and selectivity of BWCs and AWCs is completely different. Because each individual channel is small and 'invisible', it cannot be directly tested in a traditional filtration system. Instead, since BWCs are originated from biological cell membranes, they can be well characterized in artificial cells 'vesicles' that are frequently used in biophysics. In general, such transport properties are not measured in bulk but at the molecular level. Table 2 summarizes common characterization methods of biological and artificial water channels.

\subsection{Reconstitution of biological and artificial water channels and molecular transport studies}

Molecular transport properties of BWCs and AWCs can be characterized in vesicle systems. After protein purification or channel synthesis, they are reconstituted into liposomes or polymersomes by the film rehydration or dialysis methods (Fig. 5a) (Kumar et al., 2012). In the film rehydration method, a lipid or BCP film is first made after dissolving them in solvent and evaporating the solvent on a rotary evaporator at ambient temperature. The left film is further dried in a vacuum desiccator before rehydration in the buffer. The drying time is typically $1-2 \mathrm{~h}$ to overnight which depends on the volatility of the solvents used. Channel can be added at the beginning in the solvent or later during the rehydration step in the buffer with detergent stabilization as we commonly process for BWCs. When the film is rehydrated by the buffer without any detergent, liposomes or polymersomes automatically 
Table 2 Characterization methods of biological and artificial water channels

\begin{tabular}{ll}
\hline Characterization & Approach \\
\hline Overall water permeability & Osmotically induced permeability test \\
Solute rejection & \\
Channel insertion efficiency & Fluorescent correlation spectroscopy \\
Single channel water permeability & Fluorescent correlation spectroscopy + \\
Size measurement & Osmotically induced permeability test \\
Ion conductance & Dynamic light scattering \\
Water permeability of proteins/channels & Patch-clamp
\end{tabular}

Solute selectivity of proteins/channels

Dynamics of proteins/channels in bilayer membranes

Morphology of protein/channel aggregates in block copolymer/lipid membranes Transmission electron microscopy

(a)

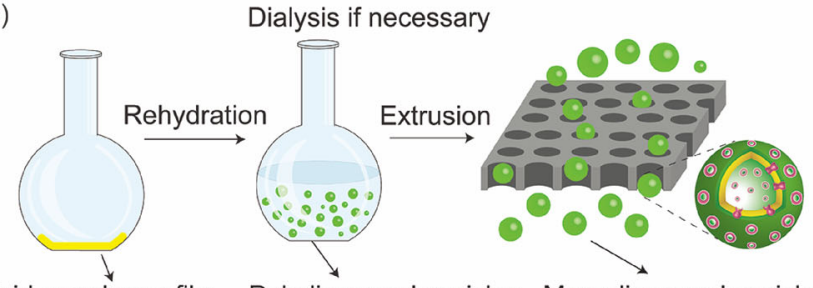

$\Omega$ Water channel $\diamond$ Water molecule, Detergent

Fluorescently tagged channel ¿ Lipid ○ Solute

Lipid or polymer film Polydispersed vesicles Monodispersed vesicles

(b)

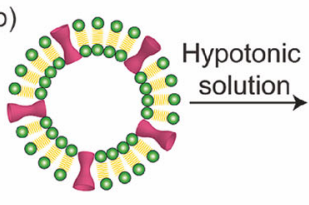

Osmotically induced water permeability measurement

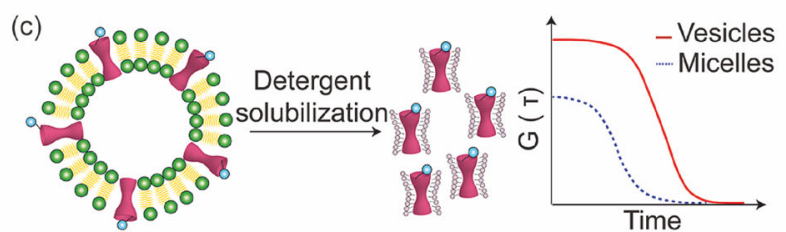

Fluorescent vesicles Fluorescent micelles Autocorrelation curves (d)

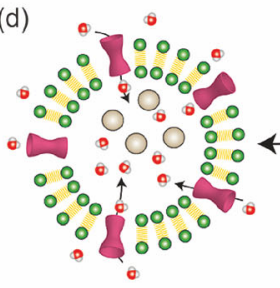

Solute is non-permeable to channels
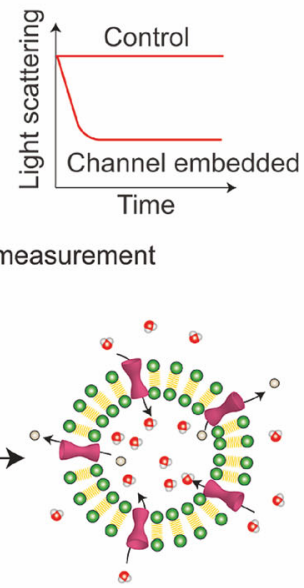

Solute is permeable to channels (e)

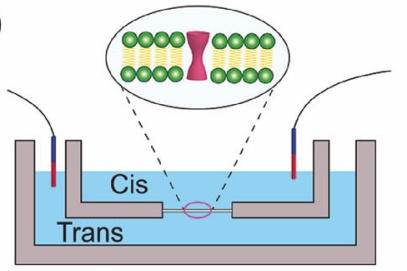

Control

Channel

A patch-clamp setup

Fig. 5 Molecular transport characterizations of biological water channels (BWCs) and artificial water channels (AWCs). (a) Reconstitution of BWCs or AWCs into liposomes or polymersomes by the film rehydration or dialysis methods. (b) Osmotically induced water permeability measurement of vesicular membranes. Vesicles swell in response to the hypotonic buffer and the light scattering at $90^{\circ}$ will decrease due to the vesicle expansion based on Reighley scattering theory. (c) Counting averaged channel number per vesicle using fluorescence correlation spectroscopy. Autocorrelation curves show a decreased intercept after the fluorophore-tagged channels are turned into individual micelles from vesicles by detergent dissolution. The ratio of the intercept before and after detergent dissolution is used to calculate averaged channel number per vesicle (see detailed description in Section 3.1). (d) Solute rejection test using osmotically induced water permeability measurement. If the channel of interest is permeable to one solute, the volumetric change upon osmotic shock will be smaller because the solute will diffuse out and compensate the volumetric change, compared to the case when using a non-permeable solute in the osmotic buffer. (e) A patch-clamp setup for ion conductance measurement.

form. If detergent exists in the system, dialysis needs to be performed to remove it from the mixed micelles to form vesicles (Rhoden and Goldin, 1979; Kaler et al., 1989; Hasler et al., 1998; Borgnia et al., 1999b; Gonen et al.,
2004; Kumar et al., 2007, 2012; Erbakan et al., 2014; Shen et al., 2015). Dialysis is performed at neutral pH (mostly 7 to 7.5) and the dialysis buffer is exchanged at an interval of $8 \mathrm{~h}$ for 3 times with a stripping to dialysis volume ratio of 
1000. The whole reconstitution process can be also completely conducted using dialysis, but the processing time will be longer. The obtained protein or channelembedded vesicles usually contain giant multilamellar vesicles and other unincorporated precipitates. The mixture undergoes a further purification step to get evenly size-distributed vesicles for subsequent analysis. They will be extruded through a polycarbonate track etched membrane (PCTE) for several times (typically $200 \mathrm{~nm}$ membrane, 10-20 times). The obtained unilamellar monodispersed vesicles with a low polydispersity index (e.g., $<0.2$ from size measurement, around $200 \mathrm{~nm}$ in diameter) are ready for osmotic permeability measurement (Kumar et al., 2012; Erbakan et al., 2014; Grzelakowski et al., 2015). Here two main bilayer systems for studying BWCs and AWCs are lipid and BCP-based bilayer membranes. The early studies on MPs used lipid membranes (Sanders and Landis, 1995; Walz et al., 1997; Hong and Tamm, 2004; Gonen and Walz, 2006; Köper, 2007; Nath et al., 2007). Bio-extracted lipids are expensive and the phosphoric head groups of these phospholipids are prone to be oxidized. Amphiphilic BCPs are an excellent alternative to lipids. The BCPs used for incorporating BWCs and AWCs are usually short in block numbers (they should be named as oligomers compared to traditional BCPs) (Zhang et al., 2012; Habel et al., 2015). They are chemically and mechanically more stable and robust, with tunable amphiphilic length and chemistry as well as a variety of functional terminal groups. The former feature is designed to be compatible with the inserted BWCs and AWCs as will be discussed in Section 4.2.1. The latter is crucial for interfacial chemistry and membrane fabrications. The most studied BCPs in this area are diblock copolymers (di-BCPs) poly(butadiene)- $b$ poly(ethylene oxide) (denoted as PB-PEO) and triblock copolymers (tri-BCPs) poly(2-methyl-2-oxazoline)- $b$ poly-(dimethysiloxane)- $b$-poly(2-methyl-2-oxazoline) (denoted as PMOXA-PDMS-PMOXA, ABA) (KitaTokarczyk et al., 2005; Kumar et al., 2007, 2012; Belegrinou et al., 2010; Malinova et al., 2010; Dorn et al., 2011; Zhang et al., 2012; 2013; Wang et al., 2013; Xie et al., 2013; Belluati et al., 2020).

Osmotically induced permeability tests are used to characterize the functionality of BWCs (e.g., AQPs) or AWCs incorporated in these vesicles (Fig. 5b). After exposed to osmotic shock (either hypertonic or hypotonic), these vesicles will shrink or expand, respectively, due to the osmotic gradient across the bilayer membranes (outward for hypertonic and inward for hypotonic). The volumetric shrinkage or expansion of the vesicles of $\sim 200 \mathrm{~nm}$ in diameter will result in the change in light scattering. Reighley scattering theory correlates the volumetric changes to the rate of light scattering change (Latimer and Pyle, 1972). After fitting the scattering trace into double-exponential functions and obtaining the kinetic coefficients $(k)$, the osmotic permeability coefficient $\left(P_{f}\right)$ of the vesicular membranes can be calculated by the following equation (Borgnia et al., 1999a).

$$
P_{f}=\frac{k}{\frac{S}{V_{0}} \times V_{w} \times \Delta_{o s m}}
$$

where $k$ is the kinetic coefficient; $S / V_{0}$ represents the vesicle surface area to initial volume; $\Delta_{o s m}$ is the difference in osmolarity between the intravesicular and extravesicular aqueous solutions and $V_{w}$ is the molar volume of water. As a result of the existence of functional channels, the vesicles with channels embedded are often shown to have faster light scattering change rate (Fig. 5b), which can be timely recorded on a stopped-flow light scattering instrument. A more accurate method is to incorporate a fluorescence dye inside vesicles as an indicator of volumetric change. In this method, the vesicles after extrusion will undergo another round purification to remove the extravesicular dyes (usually through size exclusion column or ultracentrifuge). Within proper osmolality ranges, the vesicles will act as osmometers. The relative volume (absolute volume divided by initial volume) is linearly related to the relative fluorescence (absolute fluorescence divided by initial fluorescence). The permeability can be determined in the following equation (Zeidel et al., 1992).

$$
\mathrm{d} V(t) / \mathrm{d} t=P_{f} \times S A V \times V_{w}\left[C_{i n} / V(t)-C_{\text {out }}\right]
$$

where $V(t)$ represents the relative intravesicular volume as a function of time; $S A V$ is the vesicle surface area to volume ratio; and $C_{i n}$ and $C_{\text {out }}$ are the initial concentrations of total solute inside and outside the vesicle, respectively. Other variants are the same as in Eq. (1). When permeability is measured at different temperatures, activation energy can be calculated to determine whether the water transport inside BWCs or AWCs is channelmediated (Kumar et al., 2007; Erbakan et al., 2014; Horner and Pohl, 2018).

From the above osmotic permeability measurement, what we can acquire is the overall permeability contributed by incorporated BWCs or AWCs. To obtain single-channel water permeability, the number of proteins or channels per vesicle must be known. In the early studies, this number was usually estimated by the protein to lipid ratio when preparing the samples. It may not be precise because some of the proteins and lipids may aggregate and precipitate during vesicle formation. Giant vesicles will be removed from the system during vesicle purification. All these phenomena may result in the actual protein to lipid ratio off the number as we prepared. There are two approaches to get more accurate averaged number of protein or channel per vesicle. The first method is fluorescence correlation spectroscopy (FCS) (Hoomann et al., 2013; Erbakan et al., 2014) (Fig. 5c). In FCS, proteins or channels are labeled with a fluorophore before they are incorporated into liposomes or polymersomes. The fluorescent intensity $F(t)$ 
will be recorded before and after detergent solubilization within a small confocal volume, monitored by a timeresolved single-photon counting module. These two sets of data, representing protein or channel-embedded vesicles and solubilized protein or channel micelles respectively (Fig. 5c), will create two autocorrelation functions $G(\tau)$.

$$
\begin{aligned}
G(\tau) & =\frac{\langle\delta F(t)\rangle\langle\delta F(t+\tau)\rangle}{\langle F(t)\rangle^{2}} \\
& =\frac{1}{N} \sum_{i=1}^{M} f_{i}\left[\frac{1}{1+\tau / \tau_{D_{i}}}\right]\left[\frac{1}{1+(r / z)^{2}\left(\tau / \tau_{D_{i}}\right)}\right]^{1 / 2}
\end{aligned}
$$

where $\delta F(t)$ is the fluorescence fluctuation; $t$ is time and $\tau$ represents time lag. The specific expression of $G(\tau)$ is a function of the confocal volume ( $r$ and $z$ represent the radius and half height of the confocal volume, respectively), the $2 \mathrm{D}$ lateral diffusion time $\left(\tau_{D_{i}}\right)$ of the fluorescent species $i$, and the fraction $\left(f_{i}\right)$ of fluorescent species $i$. The number of independent fluorescent molecules in the confocal volume $(N)$ can be calculated by inverting $G(0)$ when $\tau=0$. After obtaining the number of fluorescent vesicles $\left(N_{\text {vesicles }}\right)$ and solubilized micelles $\left(N_{\text {micelles }}\right)$, the number of reconstituted channels or proteins per vesicle can be calculated by the ratio of $N_{\text {micelles }} / N_{\text {vesicles. }}$. The second method is ultraviolet-visible (UV/vis) spectroscopy. Since most channels are macrocyclic molecules and can be dissolved in organic solvent, the second method is to use the specific UV/vis adsorption of the channels to estimate the final channel concentration through a calibration curve (Licsandru et al., 2016). If we know how many macrocycles compose one channel, we can calculate the channel number per vesicle. With a combination of the FCS or UV/vis data and the stopped-flow permeability data, the single-channel water permeability can be calculated (Erbakan et al., 2014; Shen et al., 2015; Licsandru et al., 2016).

Solute rejection properties can be also evaluated via the water permeability measurement. In this method, one solute will be selected as a reference (typically a solute with a large MW and regarded as not permeable to the channel of interest, e.g., Dextran500), and the water permeability in presence of other solutes will be compared to the reference solute. For example, when a hypotonic condition is applied, the inward osmotic gradient drives water molecules and small solutes to diffuse into and out of the vesicles, respectively (Fig. 5d). If the channel of interest is permeable to the solute of interest and diffuses out, the water permeability (i.e., net volumetric expansion) will be smaller due to the compensation effect compared to the permeability induced by the reference solute under the same osmolarity (Fig. 5d). This means, in the case of incomplete rejection of solutes, the apparent osmotic gradient decreases which offsets the volume change. The reflection coefficient $(\sigma)$ is calculated to evaluate the rejection properties of a channel (Meinild et al., 1998; Kumar et al., 2007; Shen et al., 2015, 2020a, 2020b; Licsandru et al., 2016; Li et al., 2019a; Song et al., 2019).

$$
\sigma_{\text {solute }}=J_{\text {Solute }} / J_{\text {Reference }}
$$

where $J_{\text {solute }}$ and $J_{\text {Reference }}$ are the volume flow rates using the selected solute, and reference solute, respectively. The ion selectivity of BWCs and AWCs can be determined using a patch-clamp setup (Fig. 5e) (Geng et al., 2014; Shen et al., 2015; Si et al., 2015; Song et al., 2020). A planar lipid bilayer membrane will be made across the aperture first. The membrane separates two chambers that are filled with different salt solutions. After the protein or channel is introduced into the lipid bilayer, the transmembrane current will be recorded and compared with the current of the native membrane. When the channel of interest is permeable to certain ions, the current of the channel-embedded membrane will be higher than that of the control membrane.

\subsection{Molecular dynamics (MD) simulations of biological}

and artificial water channels in vesicle systems

MD simulations have been demonstrated a powerful tool to assist channel studies with experiments, providing more insights into the channel dynamics within bilayer membranes, evaluating the water diffusion coefficient, water permeability and solute rejection (Shen et al., 2015, 2018; Licsandru et al., 2016; Li et al., 2019a; Song et al., 2020). Some recent protein simulation studies showed the potential to use MD simulations to guide pore engineering (Saboe et al., 2017; Chowdhury et al., 2018b). The simulations are performed using MD programs such as Nanoscale Molecular Dynamics (NAMD) (Kalé et al., 1999), and the results are analyzed and visualized by visual molecular dynamics (VMD) (Humphrey et al., 1996). Appropriate force field parameters (e.g., Chemistry at Harvard Macromolecular Mechanics, CHARMM) (MacKerell et al., 1998) and related models are selected to calculate the interactions among water, lipids, BCPs and proteins or channels. Usually, a patch of lipid or BCP membranes is first created with proteins or channels of interest embedded, or we can build and equilibrate an area of membranes and insert proteins or channels later. After the system is equilibrated, the restraints will be released. The simulation under a designated temperature, pressure and volume will be performed for a couple of hundred or thousand nanoseconds. Detailed simulation model construction can be found in these publications (Shen et al., 2015, 2018; Licsandru et al., 2016; Li et al., 2019a; Song et al., 2020).

From simulation results, we can obtain a deeper understanding of the behavior of proteins or channels in lipid or BCP membranes. First, visual snapshots can provide us a general idea about how each protein or 
channel moves within the membranes over the simulation period (Fig. 6a): if the membranes are deformed; whether the protein or channel moves out, etc. The preferable angle orientation of each protein or channel can be obtained as well. If an array of channels or proteins are simulated, we can observe the interaction among the adjacent proteins or channels and whether aggregation phenomenon would happen (Fig. 6b). This could be a good indication if the designed protein or channel can self-assemble into highly packed 2D arrays for membrane fabrication. Quantitatively, root-mean-squared deviation (RMSD) calculations are applied to quantify the dynamics of proteins or channels and the RMSD represents the configuration change of each protein or channel within the membrane (change with respect to the original coordinates) (Fig. 6c), which is indicative of the freedom of the protein or channel within the selected lipid or BCP membranes (Shen et al., 2015, 2018; Licsandru et al., 2016; Li et al., 2019a; Song et al., 2020).

MD simulations can provide transport properties of proteins or channels. The collective diffusion model is often used in the simulation under equilibrium condition to determine osmotic water permeability (Zhu et al., 2004).
First a desired volume through which water permeates should be determined, usually a region within the channel. The vector displacement along the $z$ axis of each water molecule in the region of interest within the channel will be determined. The collective displacement coordinate, $n(t)$, will be defined as the sum of the displacements divided by the length of the volume along the $z$ axis. The obtained $n(t)$ will be integrated over the simulation time $t$ for each channel. Then, the diffusion coefficient $\left(D_{n}\right)$ can be obtained by calculating the mean square displacement, $n^{2}(t)$, out of the Einstein equation:

$$
<n^{2}(t)>=2 D_{n} t
$$

The osmotic water permeability can be calculated by the following equation.

$$
p_{f}=v_{w} D_{n}
$$

where, $v_{w}$ is the average volume of a single water molecule. Additionally, water fraction within the channel and the average number of hydrogen bonds along the water wire can be estimated as well (Shen et al., 2015; Song et al., 2020). (a)

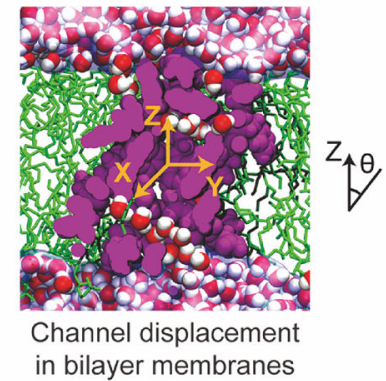

(c)

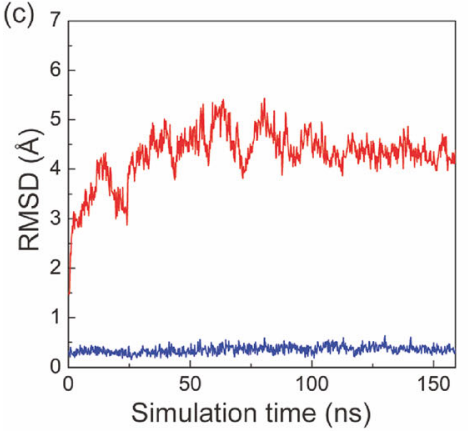

(b)

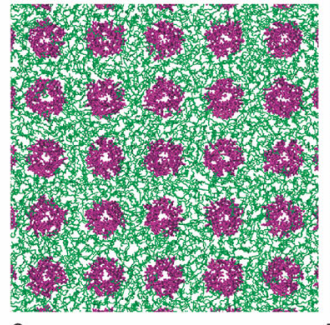

$0 \mathrm{~ns}$

(d)

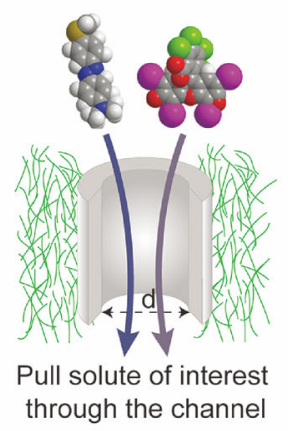

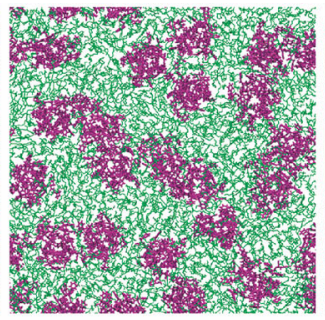

$57 \mathrm{~ns}$

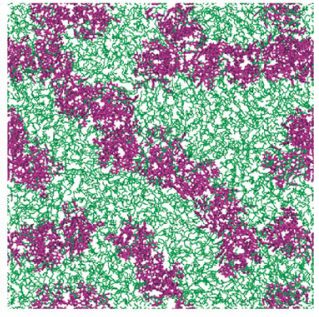

$230 \mathrm{~ns}$

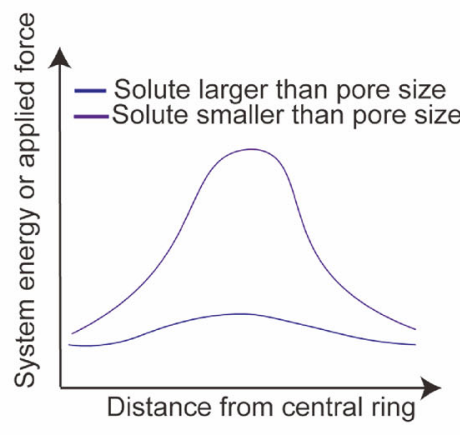

Steered MD simulations for selectivity evaluation of proteins or channels

Fig. 6 Molecular dynamics (MD) simulation of biological water channels (BWCs) and artificial water channels (AWCs) in bilayer membranes. (a) A snapshot provides the orientation of proteins or channels (e.g., peptide-appended pillar[5]arene, PAP[5]) within bilayer membranes during a simulation period. Reproduced with permission from Shen et al., 2015. Copyright 2015 National Academy of Sciences. (b) Simulation of an array of proteins or channels can help predict aggregation and the potential for self-assembly. Reproduced with permission from Shen et al., 2015. Copyright 2015 National Academy of Sciences. (c) Quantitative root-mean-squared deviation (RMSD) calculations can be used to characterize the configuration change of a protein or channel with respect to its coordination system (shown in panel a). This number can be indicative of the freedom of the selected protein or channel in the bilayer membrane. The red curve represents the RMSD of the PAP[5] channel and the blue curve represents the RMSD of the carbons in the central ring of the channel. Reproduced with permission from Shen et al. (2018). Copyright 2018 Nature Publishing Group. (d) Steered MD simulations can be used to verify the selectivity of proteins or channels. In this process, we assume to pull an atom of a solute of interest and drag it through a protein or channel. From the change in the system energy or applied force, we can determine if the solute will be rejected by the protein or channel. 
Steered MD simulations can be used to validate the selectivity of proteins or channels (Rathee et al., 2016; Shen et al., 2018). We select solutes with MWs near the cut-off of a protein or channel. Using similar force field parameters and models as mentioned above, we pull these solutes through the channel (Fig. 6d). If the selected solute is smaller than the pore diameter of the channel or has minimal interaction with the channel, the solute will pass through with no deformation, thus the applied force or the system energy will not change significantly. If the solute is too large and will cause the deformation of the pore to go through, the energy applied will increase significantly during the drag, thus implying the solute will be rejected by the channel.

\section{Biological and artificial water channel-based membranes}

After obtaining an ideal BWC or AWC, a practical question is how to use these nano-sized columnar building blocks to fabricate membranes. A random polymerization of these materials into a polymer matrix may not work since this approach does not take any advantage of the channel-dictated transport of BWCs or AWCs. A good strategy is to preserve these highly connected channel-like structures after membrane fabrication. Historically, vesicle-based membranes were made first because BWC or AWC-embedded vesicles were readily available, but the challenges were the defects and subsequent sealing brought by utilizing these spherical vesicles to make flat membranes. Highly packed and aligned BWC or AWCbased membranes with high performance were made later, but the approaches were relatively sophisticated and needed to be optimized for scaled applications. Figure 7 illustrates several stages of channel-based membranes form the discovery of AQPs in 1992 to the latest technology of channel-based membrane synthesis.

\subsection{Vesicle-based membranes}

Inspired by AQPs' marvelous water permeability and selectivity, Kumar group came up with a revolutionary idea to incorporate bacterial AQP, AqpZ, into amphiphilic BCP membranes in 2007. The AqpZ-incorporated BCP membranes could reach a permeability as high as $600 \mathrm{~L} /\left(\mathrm{m}^{2} \cdot \mathrm{h}\right.$. bar) (LMH/bar), which was about 2 orders of magnitude higher than existing RO membranes with a selectivity of $100 \%$ (Kumar et al., 2007). However, these membranes are vesicular membranes, in the form of vesicles with 200 $300 \mathrm{~nm}$ in diameter, which are different from commercial flat membranes. Over the past years, several methods have been exploited to make flat AQP-based membranes using spherical AQP-incorporated vesicles. They include 1) direct vesicle fusion, 2) charge-enhanced vesicle deposition, 3) post chemical cross-linking and 4) interfacial polymerization. Through optimizations, AQP-based membranes have overcome defect and stability issues and finally reached scalable production. The membranes fabricated by these methods are sketched in Fig. 8 and summarized in Table 3.

\subsubsection{Direct vesicle fusion}

A simple idea is to direct fuse AQP-incorporated liposomes or polymersomes onto a porous substrate (Fig. 8a). As a harbinger of this field, Freger group fused proteoliposomes onto different types of substrates. The vesicles were ruptured and formed supported lipid bilayer membranes (Kaufman et al., 2010). They found for certain substrates such as NTR-7450 NF membranes, the formation of the supported membrane was more favorable because of the charge-induced interaction between the substrate surface and lipid molecules (Kaufman et al., 2014). However, the membranes were not selective probably because of non-100\% coverage of the vesicles. Meanwhile, Wang group fused AqpZ-incorporated

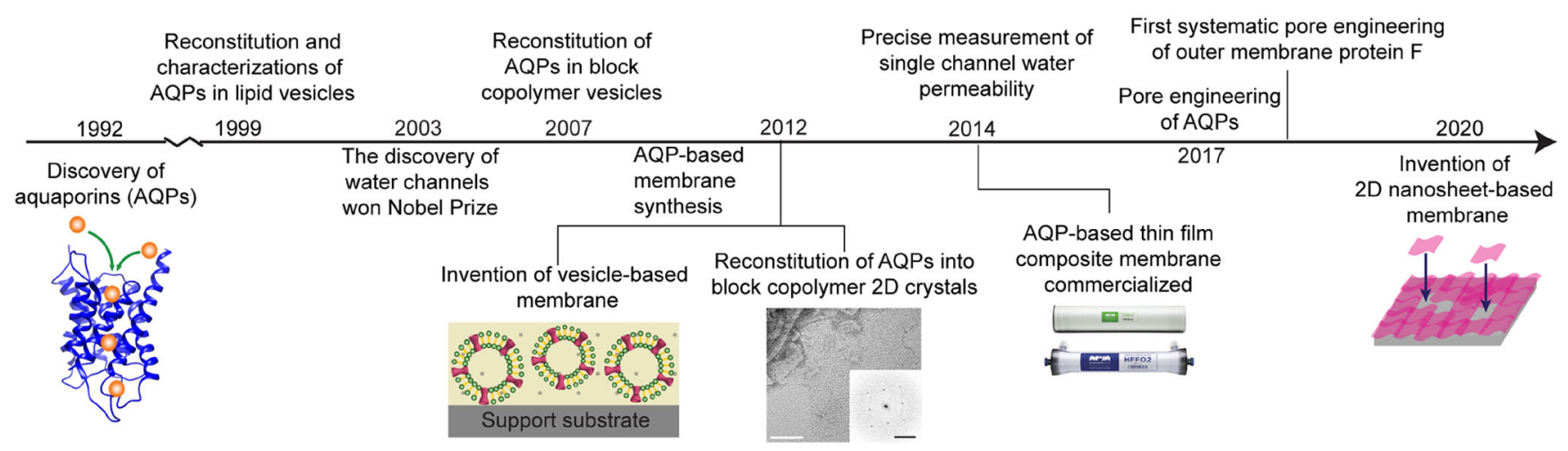

Fig. 7 Historical development of channel-based membranes. The picture of aquaporins (AQPs) 2D crystals is reproduced with permission from Kumar et al. (2012). Copyright 2012 American Chemical Society. The picture of AQP-based thin film composite membrane modules is reproduced with permission from Aquaporin A/S. 
liposomes onto NF-270 membranes (Li et al., 2012). These were the pretotype AQP-based membranes, which were in the form of supported bilayer membranes (Kaufman et al., 2010, 2014; Li et al., 2012; Wagh et al., 2015). They were frequently studied later with a spectrum of optimizations to overcome defect, stability and performance deficiencies.

\subsubsection{Charge-enhanced vesicle deposition}

A layer-by-layer method can be used to deposit AQPincorporated vesicles together with oppositely charged polyelectrolyte onto substrates (Fig. 8b). The alternate positive/negative charged layers strengthen the interlayer interaction between AQP-based vesicles and selected polyelectrolytes. Chung group fabricated an AqpZ-containing mixed matrix membrane using this method and encapsulated AqpZ-incorporated vesicles with positively charged poly-L-lysine onto polyanion films. The negatively charged liposomes were protected by the poly-Llysine layer which provided stability to the liposomes (Sun et al., 2013b). The water permeability reached $6 \mathrm{LMH} / \mathrm{bar}$ with $\mathrm{MgCl}_{2}$ rejection larger than $95 \%$. Later they created a simple method to enhance the embedding efficiency of AqpZ-incorporated vesicles in the membrane. The strategy was to incorporate magnetic nanoparticles in the AqpZincorporated vesicles in order to expedite the deposition by magnet (Sun et al., 2013a). Wang group used two polyelectrolytes (poly(ethylenimine), PEI, positively charged, and poly(sodium 4-styrenesulfonate), negatively charged) to enhance the binding between the two adjacent layers. The resulting membrane exhibited a high flux of 22 $\mathrm{LMH}, \mathrm{MgCl}_{2}$ rejection of $97 \%$ and $\mathrm{NaCl}$ rejection of $75 \%$ under an operation pressure of $0.4 \mathrm{MPa}$ (Wang et al., 2015).

(a)

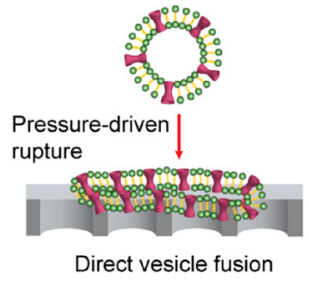

(c)

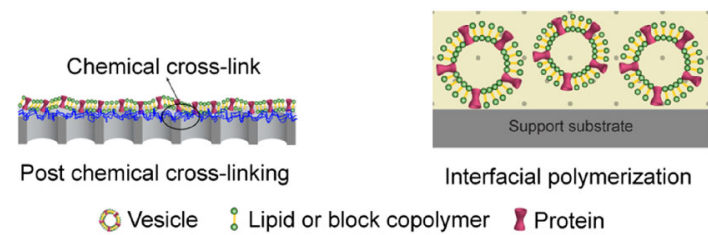

Fig. 8 Four strategies to fabricate aquaporin-incorporated vesicle-based membranes. (a) Direct vesicle fusion; (b) Chargeenhanced vesicle deposition; (c) Chemical cross-linking; (d) Interfacial polymerization.

\subsubsection{Chemical cross-linking}

Chemical cross-linking is a post-treatment after AQPincorporated vesicles are physically fused onto support membranes to form AQP-based supported biomimetic membranes (Fig. 8c). This method takes the advantages of the tunable chemistry of BCPs and supported substrates and has been systematically studied by Chung group. The cross-linking can be performed between the terminal functional groups of BCPs and the surface functional groups of the substrates to stabilize the vesicles. The ABA tri-BCPs can be functionalized with disulfide and chemically conjugated to the gold-coated porous alumina substrates through thiol chemistry (Duong et al., 2012). UV-induced methacrylate chemistry cross-linking was conducted between the methacrylate head groups of ABA tri-BCP vesicles and the acrylate residues on PCTE or silanized cellulose acetate (CA) substrates after vesicle fusion (Wang et al., 2011, 2012; Zhong et al., 2012). Using amidation reaction, hydroxyl functionalized ABA triBCPs could be cross-linked onto amine functionalized CA substrate. The synthesized membrane was found to have a well-controlled nanostructured selective layer which exhibited improved mechanical strength and stability during the water filtration process (Xie et al., 2013). In addition to the interfacial cross-linking, vesicles can be cross-linked internally to enhance the bilayer stability through methacrylate chemistry (Sun et al., 2013c). The chemical cross-linking method could be done after charge-enhanced vesicle deposition. Wang group fused and immobilized polydopamine-coated vesicles onto a poly(amide-imide) NF membrane with PEI cross-linked with the substrate (Li et al., 2014).

\subsubsection{Interfacial polymerization}

To fabricate a mechanically robust, defect-free and scalable AQP-based membrane, the traditional interfacial polymerization that is used to fabricate NF, RO and FO membranes is adopted. The idea is to embed AQP-incorporated liposomes or polymersomes into the selective layer of the thin film composite (TFC) membranes (Fig. 8d). The vesicles will be suspended in aqueous solution that contains one of the monomers $m$-phenylenediamine (MPD). After polysulfone substrates are exposed to the aqueous solution, the left MPD on the surface will react with the other monomer 1,3,5-benzenetricarbonyl trichloride at the interface and form cross-linked polyamide layer with proteoliposomes or proteopolymersomes embedded. The hypothesis is that the existence of these AQPincorporated vesicles inside the polyamide layer will provide additional water transport pathways and thus improve the water permeability and salt rejection. Because this method is established on the mature and commercialized interfacial polymerization technology, this type of 


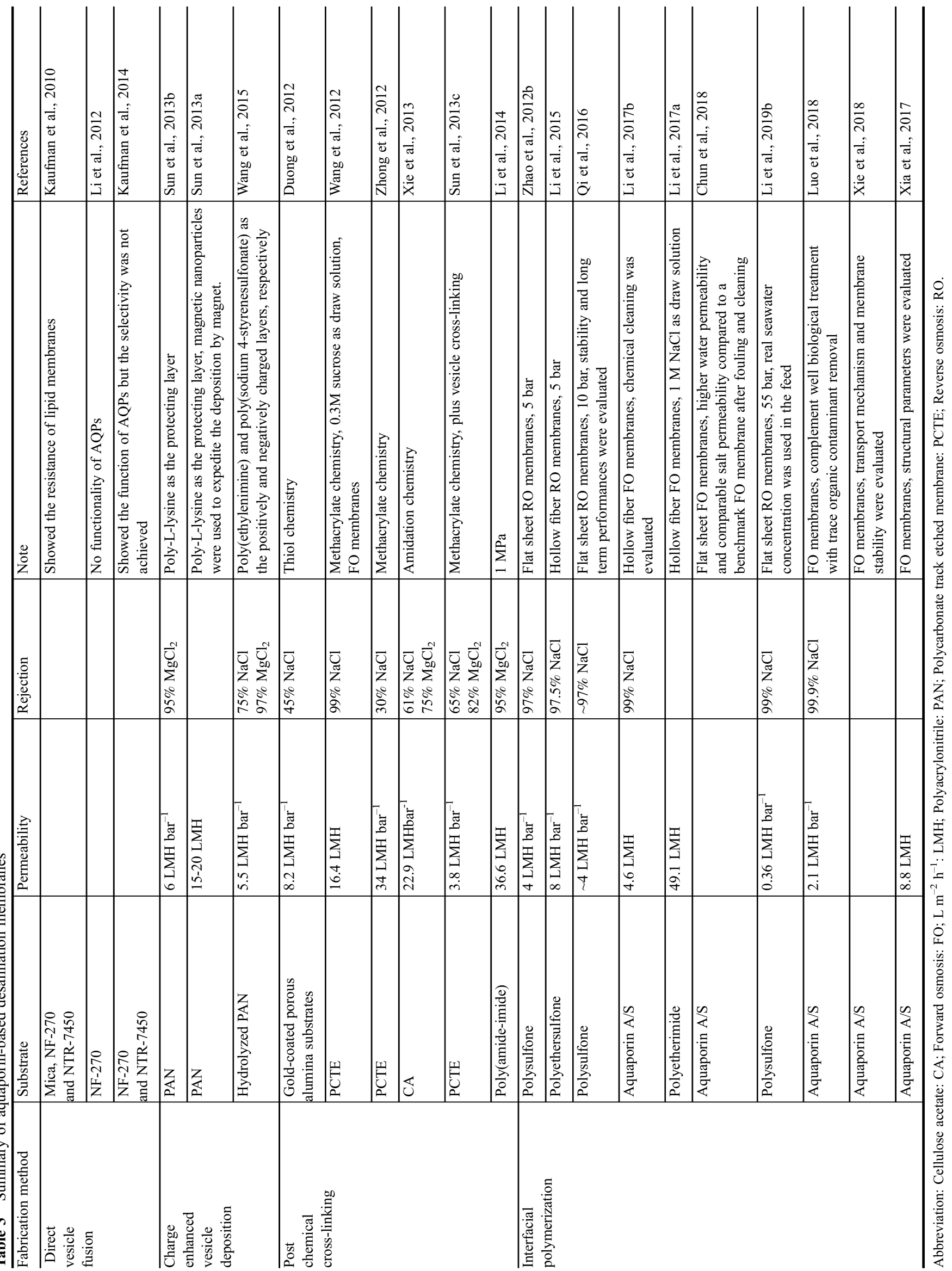


AQP-based membranes can be produced at an industrial scale. Tang group first demonstrated this concept and the membrane with AqpZ-incorporated proteoliposomes immobilized was up to $200 \mathrm{~cm}^{2}$ in size. The membrane had a similar flux compared to a brackish water RO membrane (BW30) but achieved similar salt rejection (up to $97 \%$ ) of a sea water RO membrane (SW30HR) (Zhao et al., 2012b). Later this method was validated on hollow fiber membranes, which achieved 200\% water permeability improvement and showed $97.5 \%$ salt rejection compared to BW30 (Li et al., 2015). These biomimetic membranes reduced energy input and required only half of the pressure that is applied to conventional membranes. The membranes were also tested in FO modes and no noticeable salt back diffusion was detected. Following these two proof-of-concept studies, fouling resistance, membrane stability and long-term performance were evaluated (Qi et al., 2016; Li et al., 2017b; Chun et al., 2018; Górecki et al., 2020) and highly permeable AQPbased RO and FO membranes were finally manufactured after optimizations (Li et al., 2017a, 2019b). For municipal wastewater treatment, AQP-based FO membranes were applied in an osmotic membrane bioreactor and found to well complement biological treatment in terms of notable removal efficiency of biologically persistent trace organics and have stable compatibility with activated sludge treatment (Luo et al., 2018). This type of membranes has been commercialized by Aquaporin A/S (Mentzel et al., 2014). However, the understanding of transport within the AQP-embedded polyamide layer is unclear, and the functionality of AQPs is not revealed yet. Several recent studies showed that the dominant transport was governed by the solution-diffusion mechanism (Xie et al., 2018) and the difference between AQP-based and commercially available FO membranes was not significant in terms of key parameters and performances (Xia et al., 2017).

\subsection{2-dimensional nanosheet-based membranes}

\subsubsection{2-dimensional nanosheet synthesis}

Most of BWC or AWC-based membranes adopt the aforementioned technologies: grafting BWC or AWCembedded vesicles into the selective layer of polymer membranes. This approach is straightforward, but the vesicles limit the loading density of BWCs or AWCs of interest, in addition to the stability and scalability issues. Provided cylindrical shapes of proteins or channels and their stability within hydrophobic membranes, it is possible to assemble them into $2 \mathrm{D}$ arrays with high packing density and vertical orientation. This is derived from electron crystallography several decades ago (Jap et al., 1991; Fujiyoshi, 1998). During that period, MPs were assembled into 2D arrays (i.e., 2D crystals). These biological pores are aligned vertically with high packing density epitaxially and high order within lipid membranes.
2D arrays were used for determining protein structure by electron microscopy (Jap et al., 1991; Fujiyoshi, 1998). From the separation point of view, they are ideal nanosized porous materials for molecular exclusion which enable minimum transport resistance and maximum permeation. In BWC or AWC-based $2 \mathrm{D}$ arrays, the molar lipid (or polymer) to protein (or channel) ratios are close to $\sim 1$, indicating a high protein (or channel) fraction. The packing density of $2 \mathrm{D}$ nanosheet could achieve $10^{4} \sim 10^{5}$ pores $/ \mu \mathrm{m}^{2}$ which is much higher than the density of CNTs in current CNT-based membranes (Shen et al., 2015).

2D nanosheet synthesis is similar to the recontinuation of BWCs or AWCs into vesicles (Fig. 9). Briefly, purified proteins or channels are first mixed with lipids or BCPs in detergent-added buffer and form 'mixed micelles', which will be subject to a detergent removal process. After complete removal of the detergent, 2D crystals will form under optimized conditions. The 2D nanosheets usually have micro-sized dimensions epitaxially (Fig. 9). The ordered structure can be imaged and visualized by transmission electron microscopy (TEM) or cryogenic TEM. This traditional dialysis method renders highly ordered structures but is also semi-empirical. Sometimes trial and error are necessitated to determine the optimized conditions to form 2D crystals of a particular BWC or AWC. In summary, an appropriate molar protein (or channel) to lipid (or BCP) ratio (Kumar et al., 2012; Shen et al., 2015) detergent removal rate (Kumar et al., 2012), hydrophobic physical and chemical mismatches (Ren et al., 2017; Shen et al., 2018) are the three detrimental factors for 2D nanosheet formation.

Because most BWCs or AWCs are in cylindrical shapes, insertion of these pillar-shaped blocks into amphiphilic lipid or BCP membranes with similar height will change the morphology of the formed aggregates. Most lipids or BCPs form vesicular or tubular self-assembled structures, mainly affected by their relative hydrophobic and hydrophilic block volume (Israelachvili et al., 1977; Mai and Eisenberg, 2012). With addition of BWCs or AWCs, the curvature of the formed membranes will change accordingly. These short cylindrical channels seem to flatten the membranes. As concentration of BWCs or AWCs increases, the morphology of the self-assembled aggregates transfers from monodispersed vesicle, giant vesicle, planner membrane to finally $2 \mathrm{D}$ crystals with high protein or channel packing density (Fig. 10a). This critical ratio where $2 \mathrm{D}$ arrays form needs to be determined experimentally. In 2012, Kumar group reconstituted AQP0 into two BCP membranes: di-BCP PB-PEO and tri-BCP PMOXAPDMS-PMOXA (or ABA) (Kumar et al., 2012). A clear transition from vesicles to planner membranes and tetragonal 2D crystals was observed when the molar protein to polymer ratios were increased from 0.01 , to 0.1 and $\sim 1$. The same group also tested PAP [5] AWCs in both phosphatidylcholine lipid and PB-PEO BCP membranes 


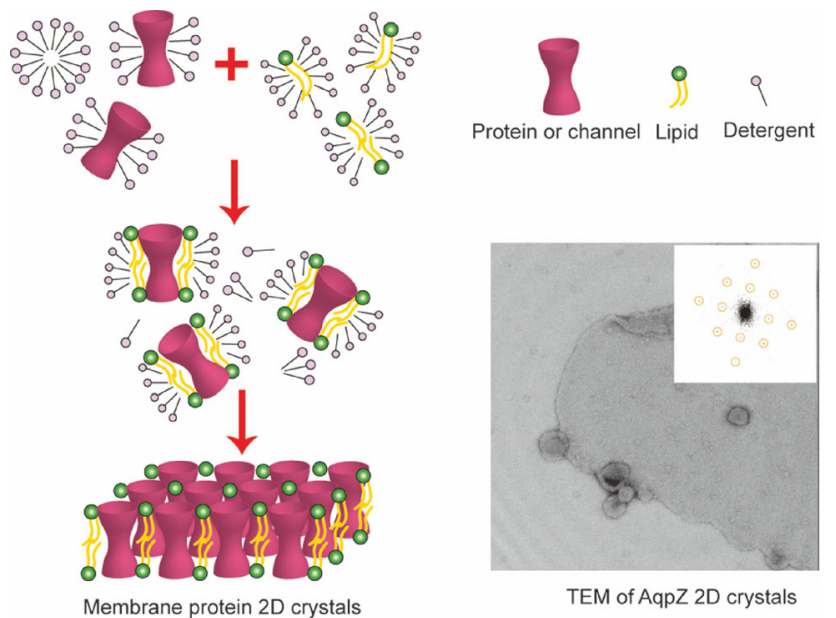

Fig. 9 The dialysis method is used to synthesize membrane protein or artificial channel-based 2D arrays. The transmission electron microscopy (TEM) image shows tetragonally packed (as indicated by the inset Fourier transform diagram) aquaporin Z (AqpZ) 2D crystals in 1,2dioleoyl-sn-glycero-3-phosphoethanolamine-N-(succinyl) lipid (unpublished data). Scale bar is $200 \mathrm{~nm}$.

(a)
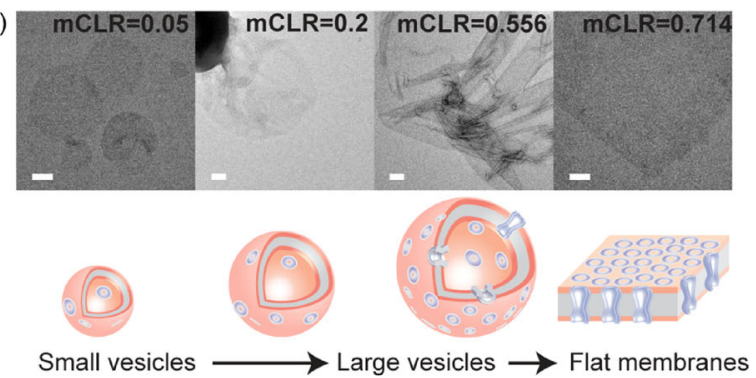

(c)

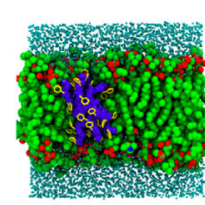

PAP[5] in POPC

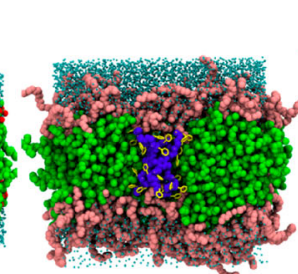

$\mathrm{PAP}[5]$ in PB12

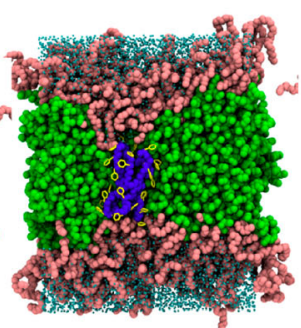

$\mathrm{PAP}[5]$ in PB23
(Shen et al., 2015, 2018). They found the similar trend and when the molar channel to lipid (or polymer) ratios were close to 1 , densely packed 2D membranes formed (Fig. 10a).

Detergent and self-assembly conditions also play important roles in protein (or channel) lipid (or BCP) aggregate morphology. To prevent any structural changes of proteins or channels from ionic amphiphiles, nonionic detergents such as octyl- $\beta, D$-glucoside (OG) are used for the dialysis process (Seddon et al., 2004). At the beginning of the dialysis, a complete dissolution of proteins or channels in detergent is needed and the dialysis usually requires $4 \%(\mathrm{w} / \mathrm{v}) \mathrm{OG}$ as the starting concentration. Since 2D array formation is thermodynamically favorable, the process kinetics (e.g., detergent removal rate and temperature) are critical. Through a series of TEM characterizations, an optimized detergent removal rate was found to be $\sim 5 \mathrm{mg} / \mathrm{ml}$ per day and this is particularly important when the detergent concentration transits through its critical micelle concentration (Fig. 10b) (Kumar et al., 2012). This process (named as slow dialysis compared to traditional dialysis) can be achieved by doubling the dialysis solution volume with detergent free buffer by every $8 \mathrm{~h}$. The
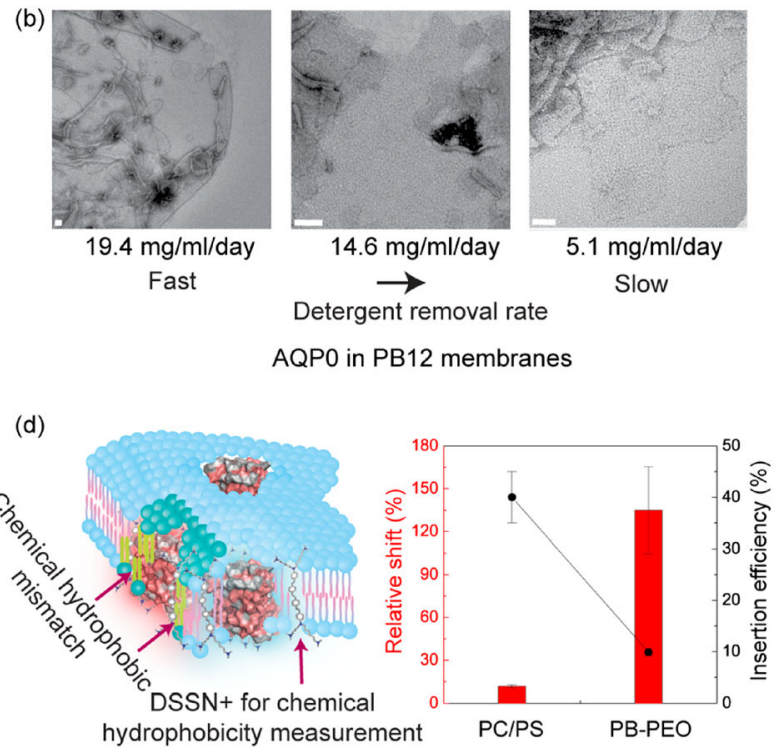

Fig. 10 Several factors influencing protein or artificial channel-based 2D array formation. (a) Protein (or channel) to lipid (or block copolymer, BCP) ratio. A clear transition from vesicular to planner membranes was observed when the molar channel (peptide-appended pillar[5]arene, PAP [5]) to lipid (phosphatidylcholine, PC) ratios (mCLR) were increased from 0.05 to $\sim 1$. Scale bars are $100 \mathrm{~nm}$. Reproduced with permission from Shen et al., 2015. Copyright 2015 National Academy of Sciences. (b) Detergent removal rate. When the detergent removal rate was lowered down to $\sim 5 \mathrm{mg} / \mathrm{ml}$ per day, aquaporin 0 (AQP0) started to form 2D crystals in poly(butadiene)- $b$-poly (ethylene oxide) (PB12) membranes when the molar protein to polymer ratio was 0.77 . Scale bars are $100 \mathrm{~nm}$. Reproduced with permission from Kumar et al., 2012. Copyright 2012 American Chemical Society. (c) Hydrophobic physical mismatch. A computational simulation showed the physical mismatch between PAP[5] channels ( 4 nm) and 1-palmitoyl-2-oleoyl-sn-glycero-3-phosphocholine (POPC, $\sim 4 \mathrm{~nm})$, PB12 $(3.7 \mathrm{~nm})$ and PB23 $(5.4 \mathrm{~nm})$ membranes and how PB23 membrane deformed around the PAP[5] channel. Reproduced with permission from Shen et al., 2018. Copyright 2018 Nature Publishing Group. (d) Hydrophobic chemical mismatch. 4,4'Bis(4'-(N,N-bis(6"-(N,N,N-trimethylammonium)hexyl)amino)-styryl)stilbene tetraiodide (DSSN+) can probe relative hydrophobicity of membrane environment. The larger relative emission shift indicates larger hydrophobic chemical mismatch. The smaller shift in PC/ phosphatidylserine (PS) membranes showed PAP [5] channels were more favorable in lipid than in PB-PEO membranes. Reproduced with permission from Ren et al., 2017. Copyright 2017 John Wiley and Sons. 
dialysis can be performed at $4{ }^{\circ} \mathrm{C}$ instead of room temperature. Kumar group found these optimizations could lower down the detergent removal rate and increase the order of the 2D crystals (Kumar et al., 2012).

Hydrophobic compatibility directly reflects the interaction between proteins (or channels) and lipids (or BCPs) and can be assessed from both physical and chemical perspectives. Physical compatibility, also denoted as physical hydrophobic mismatch, represents the difference between protein (or channel) length and lipid (or BCP membrane) thickness. For a protein or channel of interest, it is important to select a proper lipid or BCP to match the dimension in order to maximize the contact and lower the system energy for self-assembly. RsAqpZ, an AQP from Rhodobacter sphaeroides, was reconstituted into two $\mathrm{ABA}$ tri-BCPs (Ren et al., 2017). The protein had a columnar height of $\sim 3 \mathrm{~nm}$. The hydrophobic domain thicknesses of the two selected tri-BCPs were $4.4 \mathrm{~nm}$ and $9.0 \mathrm{~nm}$ for ABA22 and ABA60, respectively. The research showed that RsAqpZ had more than doubled insertion efficiency in the shorter ABA22 than the longer ABA60 membranes. PAP [5] AWCs were studied in a series of PB-PEO di-BCPs with different hydrophobic block lengths (Shen et al., 2018). PB12, PB23, and PB33 polymers had hydrophobic bilayer thicknesses of $5.1 \pm 0.6 \mathrm{~nm}, 6.0 \pm 0.5 \mathrm{~nm}$, and $7.4 \pm 0.5 \mathrm{~nm}$, while the channel had a columnar height of $\sim 4 \mathrm{~nm}$. The channel could be functionally reconstituted into PB12 and PB23 membranes, but only in PB12, this channel formed densely packed planner membranes. A computational simulation showed how PB23 membranes bent to match the dimension of the PAP [5] channels (Fig. 10c). Chemical hydrophobicity difference between proteins or channels and bilayer membranes is similar to polymer-polymer interaction described by the FloryHuggins theory (Huggins, 1942; Flory and Krigbaum, 1951; Feng et al., 2017). If the difference can be minimized, the lowered free energy of mixing can favor the insertion of proteins or channels into such membranes. This chemical difference could be quantified by a water soluble conjugated oligoelectrolyte 4,4'-bis(4'-(N,N-bis (6"-(N,N,N-trimethylammonium)hexyl)amino)-styryl)stilbene tetraiodide (DSSN+) (Fig. 10d) (Garner et al., 2010). This molecule was used to probe relative hydrophobicity of bilayer hydrophobic blocks. Insertion of DSSN+ into bilayer membranes leads to a blue shift of the emission due to the low-polarity inner core of the bilayer compared with aqueous environment (Garner et al., 2010). If the chemical mismatches between proteins or channels and bilayer membranes are larger, the relative shift after protein or channel incorporation would be larger due to more polarity change. This hypothesis has been validated using PAP[5] channels with different bilayer membranes with similar thickness (Ren et al., 2017; Shen et al., 2018). It was found that the insertion of PAP [5] channels was more favorable in lipid than in PB-PEO membranes from the perspective of chemical compatibility (Fig. 10d). The results were consistent with the finding that PAP [5] channels formed more ordered 2D arrays in lipids (Shen et al., 2015) than in PB12 membranes (Shen et al., 2018). It is critical to choose a proper lipid or BCP to match both physical and chemical compatibilities with the selected BWC or AWC in order to form highly ordered and packed 2D membranes.

Magnetic fields could be used to improve 2D crystal order. A novel method is to utilize the diamagnetic anisotropy (the intrinsic material property known as diamagnetism when a strong magnetic field is applied, as shown in Fig. 11a) of proteins or channels to impose order on the system during the dialysis-driven crystallization process. When such a magnetic field was applied during the crystallization of OmpF in PB-PEO membranes, not only the order of 2D crystals was enhanced, the size also significantly increased (Fig. 11a) (Klara et al., 2016). However, this method is not readily scalable because of the limited access to such a strong magnetic field $(7.5 \mathrm{~T})$. On the other hand, the traditional dialysis approach is the most effective way to produce high-quality 2D crystals of proteins or channels in lipid or BCP membranes. However, a large amount of expensive detergent will be consumed, and the processing time usually takes several days, which implies scalability concerns of this method. Solvent casting approach therefore may become a proper substitution to reduce the fabrication time and save chemical cost. The latest study on MP-based high-throughput filters compared both dialysis and solvent casting methods (Fig. 11b) (Tu et al., 2020). The latter approach used methanol/chloroform as solvent. When the MPs and BCP films were formed upon the evaporation of the solvent, a quick rehydration using aqueous buffer resulted in 2D nanosheet formation. Compared to the 6-day processing time of the dialysis method, the solvent method only required $2 \mathrm{~h}$ which was less time consuming and also resource efficient. However, the dialysis method produces more ordered crystal structure. Nevertheless, the successful application of the solvent method may open the possibility to scale up fabrication of channel-based membranes.

\subsubsection{Membrane fabrication}

Packed BWCs or AWCs in lipid or BCP membranes are micro-sized flakes (Fig. 12a), like other 2D materials such graphene (Guo and Dong, 2011), graphene oxide (Compton and Nguyen, 2010), $\mathrm{MoS}_{2}$ (Venkata Subbaiah et al., 2016), and Mxene nanosheets (Lei et al., 2015). A less than $100 \mathrm{~nm}$ thin layer of these 2D nanosheets immobilized on a solid porous support can reduce filtration resistance and preserve the original permeability and selectivity provided by these thousands of aligned subnanometer pores. A layer-by-layer technology, which is the technology to build up multilayer constructs using different materials, was successfully modified and adopted to make 2D nanosheet-based membranes (Rajesh et al., 2014). This method was first reported in the study of PAP 
(a)
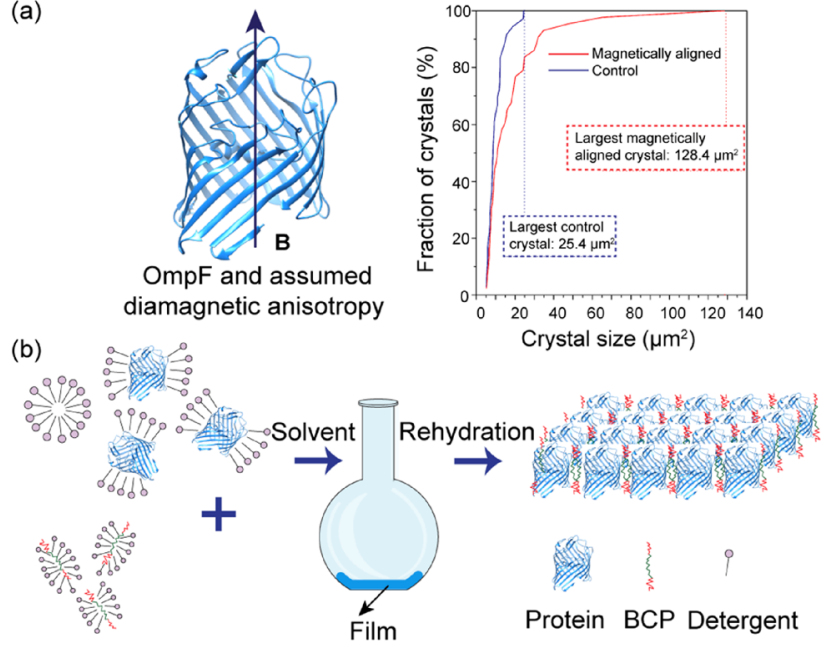

Fig. 11 Two approaches to optimize membrane protein or channel-based 2D crystal formation. (a) Under a magnetic field, outer membrane protein $\mathrm{F}(\mathrm{OmpF})$ was expected to have a preferred orientation and the $2 \mathrm{D}$ crystals formed in block copolymer (BCP) membranes had bigger size and higher order. Reproduced with permission from Klara et al. (2016). Copyright 2016 American Chemical Society. (b) The solvent method is used to synthesize membrane protein or artificial channel-based 2D arrays in $\mathrm{BCP}$ membranes within a few hours.

[5] AWC-based membranes (Fig. 12a) (Shen et al., 2018). The 2D nanosheets were made by the slow dialysis method (Section 4.2.1) (Kumar et al., 2012). To enhance the interaction between the 2D nanosheets and interlayered cationic polyelectrolyte PEI, carboxyl-functionalized
BCPs were used to ensure the final 2D sheets were fully carboxyl-terminated on both sides, which fully took the advantage of the chemical turnability of BCPs (Feng et al., 2017). A PCTE membrane with $50 \mathrm{~nm}$ pore and a polyethersulfone membrane with $30 \mathrm{~nm}$ pore were applied as the substrates, respectively. Before layer-by-layer deposition, the support membranes were treated in a UV/ ozone cleaner to obtain a negatively charged surface. The cleaned membranes were then assembled into a stirred cell covered with cationic PEI. Some of the polymers formed electrostatic interaction with the oppositely charged base membranes. The membranes were rinsed with deionized water and the extra PEI was washed off. In the next step, a solution of PAP[5] 2D nanosheet suspension was loaded on the top of the substrate and the nanosheets were physically deposited onto the substrate by filtration. Multiple rounds of PEI/PAP[5] nanosheets deposition were conducted until the surface was observed with no defects, which could be tracked by scanning electron microscopy (Fig. 12b). It was found 3 to 4 rounds of deposition could ensure $\sim 100 \%$ coverage of the nanosheets on the substrates. After the physical deposition, the PEI and carboxyl-functionalized BCPs were cross-linked using 1-ethyl-3-(3-dimethylaminopropyl)carbodiimide hydrochloride to enhance the mechanical strength of the composite membranes. Following the similar procedure, Tu et al. 2020 employed $3 \beta$-barrel BWCs (OmpF, FhuA $\Delta \mathrm{C} / \Delta 4 \mathrm{~L}$ and $\alpha \mathrm{HL}$ ) instead of AWCs and developed BWCbased membranes (Tu et al., 2020). The membranes from three selected BWCs have pore sizes of $0.8 \mathrm{~nm}, 1.3 \mathrm{~nm}$, and $1.5 \mathrm{~nm}$, respectively. In this study, the $2 \mathrm{D}$ nanosheets (a)

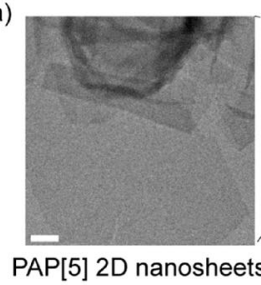

(b)

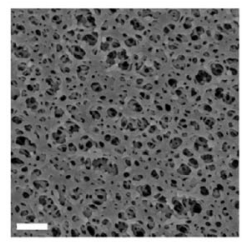

PES substrate

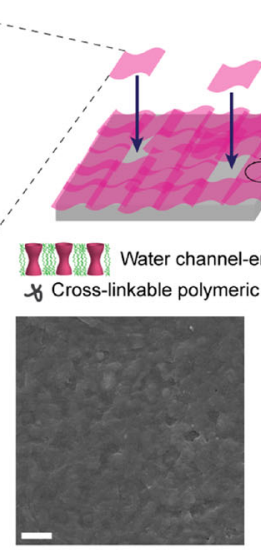

PES covered with 2D nanosheets

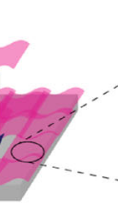

(t) 6 $10 \%$ . $\int_{0}^{0} \int_{0}$

osheet $\mathrm{H}_{2} \mathrm{O}$

出 Rejected solute

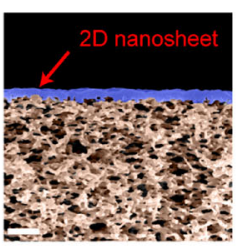

Cross-sectional view (c)

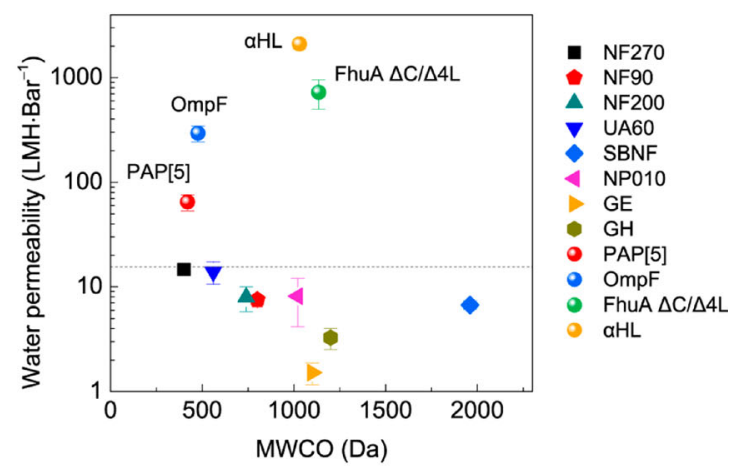

Fig. 12 A modified layer-by-layer technique for synthesis of membrane protein (MP) or channel-embedded 2-dimensional (2D) nanosheet-based membranes. (a) A schematic illustration of layered deposition of 2D nanosheets onto a porous support. The transmission electron microscopy image (unpublished data) showed peptide-appended pillar[5]arene (PAP[5]) formed 2D nanosheets in poly (butadiene)- $b$-poly(ethylene oxide) $\left(\mathrm{PB}_{12}-\mathrm{PEO}_{10}\right)$ membranes. Scale bar is $200 \mathrm{~nm}$. (b) Scanning electron microscopy images (unpublished data) showed the polyethersulfone (PES) support before and after PAP [5]-embedded 2D nanosheet immobilization, and the cross-sectional view showed the thin layer of the $2 \mathrm{D}$ nanosheets. Scale bars are $2 \mu \mathrm{m}$. (c) Comparison of water permeability (LMH/bar) and molecular weight cut-off (MWCO) (Da) of MP or channel-embedded 2D nanosheet-based membranes with commercial nanofiltration (NF) or ultrafiltration membranes. Reproduced with permission from Tu et al. (2020). Copyright 2020 Nature Publishing Group. 
were prepared by both dialysis and solvent methods (Section 4.2.1); the latter approach accelerated the processing time significantly.

The 2D nanosheet-based membranes are endowed with the inherent permeability and selectivity of the embedded BWCs or AWCs (Fig. 12c). The permeabilities of PAP [5], OmpF, FhuA $\Delta \mathrm{C} / \Delta 4 \mathrm{~L}$ and $\alpha \mathrm{HL}$-based membranes were $65 \pm 13,293 \pm 51,725 \pm 226$ and $2,107 \pm 235 \mathrm{LMH} /$ bar, with MWCOs of $\sim 450, \sim 480, \sim 1,130$ and $\sim 930$ Da, respectively (Shen et al., 2018; Tu et al., 2020). Within the comparable molecular exclusion range (500 to $1000 \mathrm{Da})$, these biomimetic membranes demonstrated 1 to 3 orders of magnitude higher permeability compared to commercial membranes. A more comprehensive characterization of the $d$-spacing between the lamellar 2D sheets by X-ray diffraction indicated $\sim 1.5 \mathrm{~nm}$ spacing and lateral flow between the adjacent layers, but the solute rejection was from the vertical size exclusion through the embedded channels (Shen et al., 2018).

\subsection{Lamellar block copolymer channel-based membranes}

Although BWCs and AWCs have been successfully employed for membrane fabrication and showed their intrinsic selectivity and permeability, the aqueous-based processing (i.e., aqueous-based self-assembly and the modified layer-by-layer technique) is not suitable for scale-up. The membrane area is limited by the size of the 2D nanosheets. The processing time is relatively long. Even if the solvent casting approach is adopted, it may still require approximately one day including nanosheet transfer, immobilization and cross-linking. To optimize the fabrication process and make this type of membrane more scalable, solvent-based process is needed. Taking the advantage of solvent compatibility of both AWCs and BCPs, lamellar BCP channel-based membranes can be made with less complicated steps and processing time (Fig. 13a) (Lang et al., 2018). The hypothesis of this approach is that biological channels or AWCs will automatically insert into the hydrophobic region of the lamellar BCPs which are reminiscent of lipids, with alternating hydrophilic and hydrophobic layers. With optimized design, the inserted channels can align and interact well with the hydrophobic domains due to the channel's hydrophobic shell and favored hydrophobic interaction.

To test this hypothesis, a new series of poly(isoprene)- $b$ poly(ethylene oxide)- $b$-poly(isoprene) (IOI) tri-BCPs were designed where 'I' represented the cross-linkable hydrophobic block poly(isoprene) and ' $\mathrm{O}$ ' represented the hydrophilic block poly(ethylene oxide) (Lang et al., 2019). First IO di-BCPs were synthesized and tested to ensure a low background water permeability using vesiclebased transport studies, indicating membranes made of this type of BCPs can act as a non-porous substrate. Then IOI tri-BCPs were designed to fabricate lamellar membranes (Fig. 13b). Briefly, a water-soluble sacrificial polymer layer poly(3,4-ethylenedioxythiophene) polystyrene sulfonate (PEDOT:PSS) was spin coated on a UV/ozone treated silicon wafer. IOI tri-BCP (with or without channels) solution in tetrahydrofuran, together with the photoinitiator and cross-linker, was spin coated on the top of this PEDOT:PSS layer. After the film was cross-linked under UV by thiol-ene click chemistry, it can be detached from the substrate by immersing the wafer into water carefully to dissolve the sacrificial layer. The floated film was later transferred onto another substrate for characterizations. The first IOI-based membranes showed lamellar structures, but also contained micro-sized defects due to the semicrystalline property of PEO domain covering the entire polymer film. After suppressing this effect using lithium bis(trifluoromethanesulfonyl)imide (LiTFSI) during film
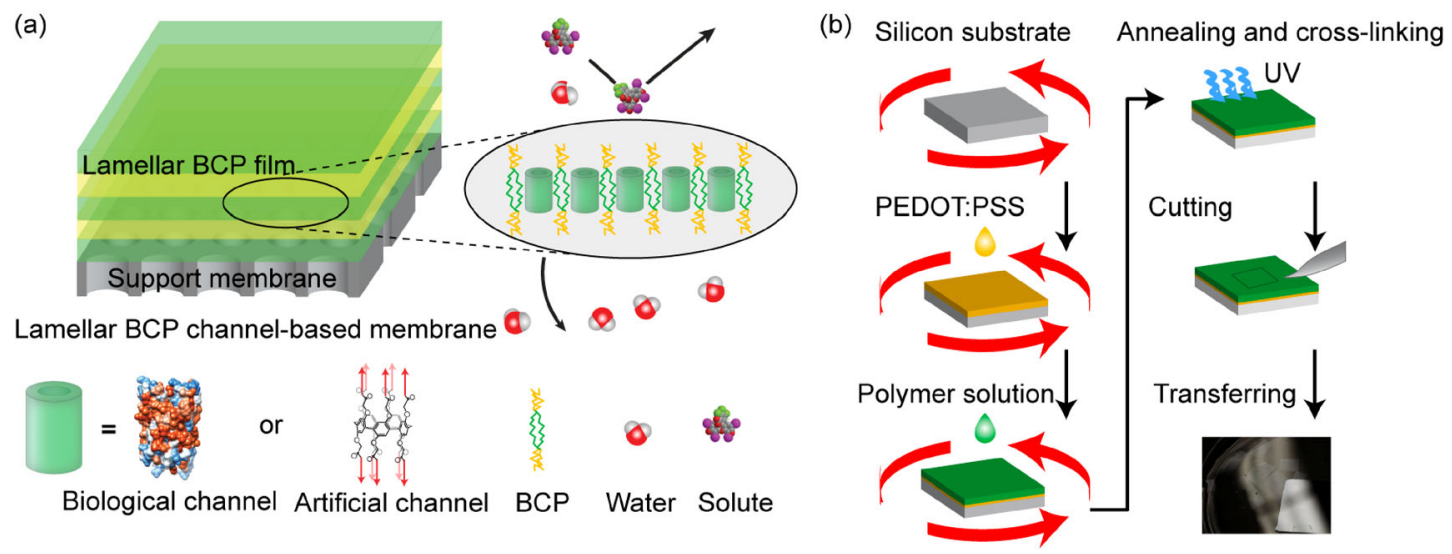

Fig. 13 Fabrication of lamellar block copolymer (BCP) channel-based membranes. (a) A schematic illustration of the design of lamellar BCP channel-based membranes. Reproduced with permission from Lang et al. (2019). Copyright 2019 American Chemical Society. (b) A schematic illustration of BCP film fabrication using a spin coating technique. 
formation (Benrabah et al., 1993), the optimized LiTFSIdoped film showed defect-free 3-layer lamellar structures, as demonstrated by atomic force microscopy and grazing incidence small-angle X-ray scattering. The insertion of fluorophore-labeled PAP[5] channels was confirmed by confocal microscopy.

The optimized film was transferred onto an aluminum oxide substrate to form a TFC membrane, with approximately $90 \mathrm{~nm}$ thickness on the top of the substrate. The control membrane, after additional sealing procedures, showed a very low background permeability below 1 LHM/bar. After PAP[5] channels were embedded, the permeability improved by $\sim 6$ fold and the membranes displaced a sharp MWCO transition from 350 to $500 \mathrm{Da}$, which was characteristic for PAP [5] and consistent with the previous molecular transport studies in lipid vesicles and nanosheet-based membranes ( $450 \mathrm{Da})$ (Shen et al., 2015,2018 ). This IOI tri-BCP-based lamellar membrane can be used as a general scaffold membrane for other functional channels such as gramicidin A to fabricate channel-based ion exchange membranes.

\section{Engineered biological and artificial water}

\section{channels}

Pore engineering is necessary in order to improve the permeability and selectivity of existing BWCs and AWCs. Modifying the internal environment of biological pores can be achieved through site-directed mutagenesis assisted by computational simulations. Traditional post modifications are difficult because of the complexity of these transmembrane pores. For synthetic channels, a systematic pore engineering is not well established. The modification methods were specially tailored for a particular type of channels.

\subsection{Pore engineering of biological water channels}

Modifying pore structures of existing BWCs can improve their permeability and selectivity. AQP0 is a water channel protein that is exclusively expressed in lens (Virkki et al., 2001). Compared to other members of the ubiquitous AQPs family such as AQP1 which mainly exists in red blood cells and kidney (Sabolic et al., 1992), the permeability of AQP0 is much lower (Agre, 2004). This is because in AQP0, the two pore-lining amino acid residues, Tyr23 and Tyr149 extend toward the pore center and lower the water permeability (Fig. 14a). In AQP1, the corresponding residues are substituted by smaller Phe24 and Thr157, respectively (Saboe et al., 2017). Two single mutants AQP0-Y23F and AQP0-Y149T and one double mutant AQP0-Y23F/Y149T were constructed to verify the steric effect hypothesis (Saboe et al., 2017). The double mutant elevated the permeability by 40 -fold to a level comparable to that of AQP1. The simulation results revealed Tyr23 was more dominant for the low water permeability of AQP0 rather than Tyr149. The mutation of Tyr23 also changed the pore profile at the near-by gate formed by residue $\operatorname{Arg} 187$.

Following this research, a systematic angstrom-scale pore engineering was conducted using a workflow combined with PoreDesigner, MD simulation and experimental validation (Fig. 14b). The study selected OmpF, a $\beta$-barrel protein with a pore diameter of $\sim 0.8 \mathrm{~nm}$, as the engineering platform (Chowdhury et al., 2018b). The native $\mathrm{OmpF}$ has a much higher single-channel water permeability compared to AQP1, without any salt rejection (a corresponding $\mathrm{MWCO}$ of $\sim 600 \mathrm{Da}$ ). To engineer $\mathrm{OmpF}$ to maintain its high water permeation while rejecting salt, the single-file water wire from AQP1 was first constructed from $\mathrm{MD}$ simulations, used as a template and computationally displaced into the lumen of OmpF (Fig. 14b). The PoreDesigner leveraged iterative protein design algorithm to computationally mutate the pore-lining amino acid residues to fill up the void spaces around the water wire. The design enabled minimum interaction between the water wire and pore wall and used relatively large hydrophobic amino acids such as phenylalanine and tryptophan to shrink the pore size. After simulations, three representative mutant configurations were created: uniform pore closure design (UCD), off-center pore closure design (OCD) and cork-screw design (CSD) (Fig. 14b). The representative mutant of each category was selected, expressed and purified. They were reconstituted into liposomes for permeability and selectivity characterizations experimentally. The stopped-flow lightscattering measurement combined with FCS determined that the permeabilities of all these three mutants $((3.3 \pm 0.2)$ to $14.7 \pm(0.9) \times 10^{10}$ water molecules per second) were 1 order of magnitude higher than that of AQP1, and also higher than the native OmpF due to the minimized waterpore wall interactions as designed. This mechanism was further confirmed by subsequent MD simulations. The solute rejection tests showed MWCOs were $\sim 342 \mathrm{Da}, \sim 180$ $\mathrm{Da}$ and $\sim 58 \mathrm{Da}$, for OCD, CSD and UCD, respectively. The UCD configuration was an AQP-like engineered pore that had higher water permeability and achieved salt rejection. Using this approach, it is possible to tune any subnanometer pores for a variety of desired molecular separations. Practical challenges of using biological channel proteins are protein expression and structural stability. The mutant may result in a low-yield expression and the structure may not be stable as the native protein.

\subsection{Pore engineering of artificial water channels}

AWCs have been improved compared with their ancestors upon structure, permeability and selectivity. 1) Pore 
(a)

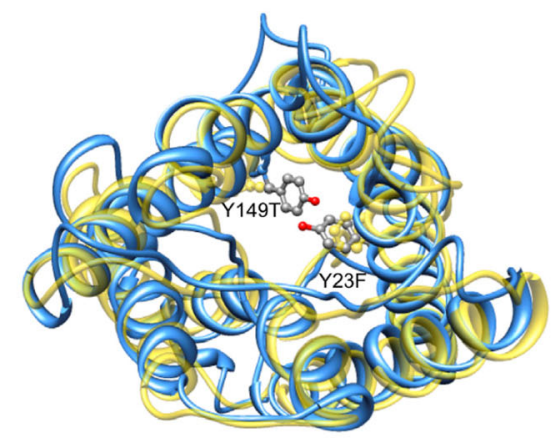

Structural comparison between AQP0 and AQP1 (b)

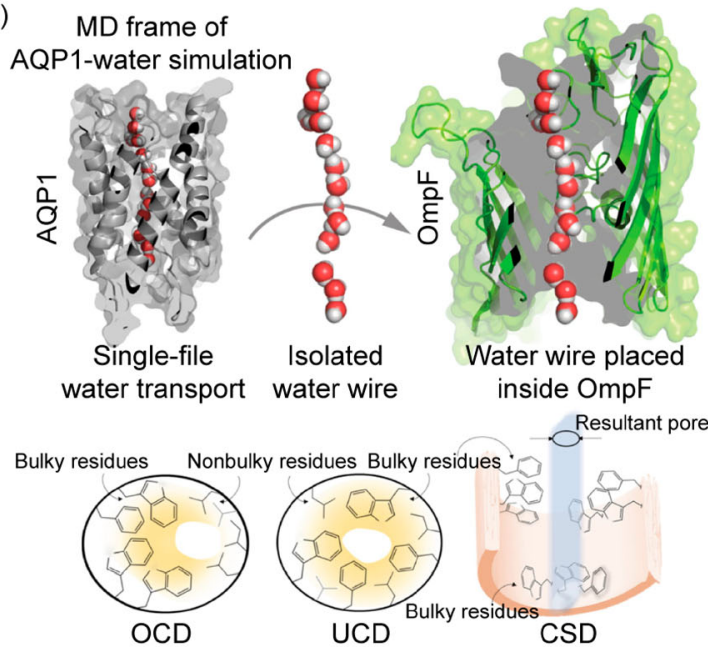

\begin{abstract}
Fig. 14 Pore engineering of biological water channels. (a) Structural comparison of low-water-permeability aquaporin 0 (AQP0, blue ribbon) and high-water-permeability aquaporin 1 (AQP1, yellow ribbon) shows Tyr23 and Tyr149 of AQP0 (shown in gray with red hydroxyl groups) extend into the water pathway, which is the reason for the low water permeability of AQP0. Reproduced with permission from Saboe et al. (2017). Copyright 2017 Elsevier. (b) PoreDesigner was used to reengineer the pore of outer membrane protein F (OmpF) to make it have AQP-like permeability and salt rejection properties. First, a water wire from AQP1 generated by molecular dynamics (MD) simulation was placed in the lumen of OmpF. It was used as a template to redesign the OmpF pore geometry by filling up the empty space around the water wire using large hydrophobic amino acids as the pore-lining residues. The mutations have three representative internal pore geometries: off-center pore closure design (OCD), uniform pore closure design (UCD), and cork-screw design (CSD). Reproduced with permission from Chowdhury et al. (2018). Copyright 2018 Nature Publishing Group.
\end{abstract}

environment becomes more hydrophobic, with less or selected hydrogen bonds. This can be seen from hydrazideappended pillar[5]arenes (Hu et al., 2012) to PAP[5] channels (Chen et al., 2013; Shen et al., 2015, 2018). The latter design used more hydrophobic amino acids thus reducing the hydrogen bonds between the pore wall and encapsulated water wire. 2) Pore size also becomes narrower to effectively reject salt. Pillar[5]arenes were too large to occlude salt therefore a smaller hybrid[4]arene ring was adopted (Song et al., 2020). Zeng group optimized the pore sizes of their aquafoldamer channels within the range of 3-4 $\AA$ to achieve a good balance between permeability and selectivity (Shen et al., 2020b). 3) Water wire becomes more ordered. The imidazole channels designed by Barboiu group (Licsandru et al., 2016) and the latest pillar[5]arene channels designed by Wang group ( $\mathrm{Li}$ et al., 2019a) both used the chiral ideas to improve the order of encapsulated water molecules.

However, systematic pore engineering has not been demonstrated so far and seems to be difficult for AWCs. The obstacles mainly come from available channel scaffolds, synthesis and post modifications. Most biological channels (e.g., AQPs and $\mathrm{OmpF}$ ) are asymmetric. Sometimes one pore-lining amino acid mutation can effectively change the pore size and selectivity, which can be achieved by well-established site-directed mutagenesis. This approach may not be directly adopted for AWCs, which mostly have symmetric designs (e.g., pillar[5]arene channels) or are self-assembled by repeatable subunits (e. g., aquafoldamer channels). Pore engineering of AWCs can be learned from other porous materials such as porous organic cages (Hasell and Cooper, 2016), metal organic frameworks (MOFs) (Cohen, 2012; Kalaj et al., 2020), and covalent organic frameworks (COFs) (Nagai et al., 2011). In most cases, symmetric post modifications are used. Asymmetric synthesis or post modifications can be extremely difficult, which have been demonstrated successful in other porous materials such as porous organic cages involving multiple protection and deprotection steps (Liu et al., 2019b). The payback of the modified cage is a highly tailored selective material for valuable hydrogen isotope separation. Theoretically, this approach is doable, but will significantly increase the cost. Take pillar[5]arene channels as an example, the five side chains are symmetric on both sides of the macrocyclic ring. It will be considerably tough to modify one side chain while protecting the others which could lead to a very low synthesis yield. When employing the methodologies from other porous materials, we need to consider the uniqueness of AWCs as well: 1-dimensional structures vs. 3-dimensional porous organic cages, MOFs and COFs, etc. With the assistance from inspiration of biological systems (Sanchez et al., 2005; Liu et al., 2013, 2017; Wegst et al., 2015), synthesis breakthrough (Tabushi et al., 1982; Lehn, 1988; Lehn, 1990), computational simulation (Böckmann et al., 2008; Hub et al., 2009; Kutzner et al., 2011), and robotic experimental platform (Burger et al., 2020), future AWCs can be more delicate and selective. 
However, merits coexist with drawbacks: performance $v s$. cost, which will be discussed in the final section.

\section{Challenges and outlook}

6.1 Scalability and practicability of current biomimetic membranes

Biomimetic membranes have been through two stages: 1) AQP-based membranes, 2) BWC or AWC 2D nanosheetbased membranes and lamellar BCP membranes. The first step of biomimicry was to directly utilize these biological building blocks for membrane fabrication. After demonstration in the laboratory-scale studies for several years, Aquaporin A/S has successfully commercialized AQPbased membranes. They immobilize vesicles incorporating AQPs in the selective layer of current RO or FO membranes. This is a benchmark showing that biomimetic membranes can be applied in industry. However, questions regarding the additional cost from protein purification, functionality of AQPs within the polymer membranes, long-term stability and safety issues still exist (Holme et al., 2015).

The second stage of biomimetic membranes is mimicking AQPs and building synthetic analogs of AQPs. A variety of artificial structures (i.e., AWCs) and related membranes have been developed around these materials. These membranes have shown the inherent properties of the embedded BWCs or AWCs, as a proof of concept that nano-scale structures inspired by nature determine membrane performances. The 2D nanosheet-based membranes have high permeability, up to 3 orders of magnitude enhancement in contrast to comparable commercial NF membranes. The MWCOs are much sharper as well. This is due to the densely packed BWCs or AWCs per unit area $\left(\sim 10^{4}-10^{5}\right.$ pores $\left./ \mu \mathrm{m}^{2}\right)$. However, such high permeability is not necessary since selectivity is more critical. The concentration polarization effect near membrane surface will offset the benefit from permeability enhancement (Werber et al., 2016a; Shi et al., 2017; Abdulsalam Ebrahim et al., 2020). Several previous studies and analysis have shown that approximately 1 order of magnitude permeability enhancement are reasonable considering both capital and operational costs (Okamoto and Lienhard, 2019; Nassrullah et al., 2020). The loading of BWCs or AWCs within biomimetic membranes are needed to be optimized to balance permeability and material cost. However, the layer-by-layer technology is difficult to scale up. The size of this type of membranes is small because of the micro-sized 2D nanosheets. The utilization of lipids or BCPs as matrix membranes also increase the fabrication cost. These materials are not only expensive but will result in poor rejection of hydrophobic solutes (Werber and Elimelech, 2018). If lipids or BCPs are mainly used as separation layer matrix, seawater desalination and ultrapure water production are appropriate applications. Wastewater reuse may not be suitable since lipid or BCP membranes cannot sufficiently reject small neutral hydrophobic contaminants such as pharmaceutical compounds. Additionally, although AWCs are considered more robust and stable than AQPs, these chemicals are still in the development stage, with multiple-step synthesis and relatively low yields.

Solvent casting has been used to make lamellar BCP biomimetic membranes. This method is adopted from the thin film fabrication technique in semiconductor industry. The processing time can be reduced significantly. Compared to $2 \mathrm{D}$ nanosheets, the packed channels in these lamellar membranes are diluted. The membranes are also restricted to smaller sizes compared to conventional polymeric membranes. However, this approach can produce scalable membranes for certain applications if optimized properly. It is important to note that currently most available BWCs and AWCs (except AQPs) used for membrane fabrication are not strict water channels. The selectivity locates within NF range and the mechanism is exclusively size exclusion. Within this range (i.e., angstrom-scale separations) lies a variety of critical applications. We believe artificial channel-based highly selective membranes will benefit this area.

\subsection{Future direction of bioinspired and biomimetic} membranes

The ultimate goal of biomimetic membranes is to borrow ideas from nature-evolved highly efficient and selective transport across cell membranes, re-innovate traditional separation membranes and bestow them with these properties. With more and more chemicals produced today and to be produced in the future, we are facing separation challenges as current membranes technologies may reach their limits (e.g., the permeability-selectivity trade-off) (Robeson, 1991, 2008; Freeman, 1999; Shannon et al., 2008; Greenlee et al., 2009; Elimelech and Phillip, 2011; Guo et al., 2012; Adewole et al., 2013; Sholl and Lively, 2016; Werber et al., 2016b; Koros and Zhang, 2017; Park et al., 2017). This is particularly important for angstrom-scale separations. Within this range, a number of critical and rewarding applications exist: pharmaceutical and antibiotics separations (Harrison et al., 2015; Li et al., 2020), extraction and recovery of highly valuable metals including light lithium (Liu et al., 2019a), heavy metals (Barakat, 2011; Pollmann et al., 2016; Falagán et al., 2017), noble metals and rare earth elements (Hodnik et al., 2016; Yang et al., 2017; Balaram, 2019; Cheisson and Schelter, 2019), oil and gas refinery (Peng et al., 2018), gas separations (Dalane et al., 2017), separation of challenging environmental co-contaminants (Madsen et al., 2015; Miao et al., 2020) (such as 1,4-dioxane and coexisting 
chlorinated solvents that are similar in size and shapes). All these applications involve filtrate molecules with similar dimensions, polarities or charges from a few hundred to several thousand Dalton. Biomimetic membranes as described in this review have shed lights on pore engineering for angstrom-scale separations. Precise design of pore structures at nanoscales allows molecular exclusion in light of steric hindrance, electrostatic repulsion, dielectric effect and van der Waals force instead of the solution-diffusion mechanism (Epsztein et al., 2020). In this regard, bioinspired separation materials and other porous materials such as MOFs, COFs, porous organic cages and 2D materials (Qiu et al., 2014; Liu et al., 2016) tread different paths that lead to the same destination: engineering membrane structure and transport at molecular scales, thus improving the overall separation efficiency. They are also faced with similar challenges and follow the Gartner Hype Cycle (Fig. 15a) (McCutcheon, 2019). New technologies usually flourish rapidly after invention, and then peak at a point of 'inflated expectations' before falling quickly into a 'disillusion valley' when the technologies fail to deliver the promises. These technologies may recover and eventually find their own niches and reach some level of commercial success. Most academic research overlays well with this cycle, accompanied with tons of publications, presentations and funding opportunities at the beginning before vanishing in our eyesight shortly. These novel materials possess remarkable separation efficiencies but are difficult to be integrated into traditional scalable membrane industry. The market is there (i.e., a variety of challenging separations and valuable products). In this regard, how to develop highly selective pore structures and transplant them into scalable membranes are two major challenges from fundamental and practical perspectives, respectively.

We first need breakthroughs in fundamental science. The key for pore engineering from the biomimetic perspective is discovering nature-evolved, highly efficient biological transport channels and developing synthetic approaches that can precisely mimic those transports. Fundamental understanding at the molecular level and high-yield and scalable synthesis technologies are imperative. Following the above steps is to engineer the nanoscale structures of separation membranes, which usually employ these biological or synthetic building blocks to make highperformance membranes as a proof of concept and validate the hypothesis.

After those new concepts are triggered, we need to bridge fundamental and applied researches. The key for separation science is to bridge novel and traditional separation materials. There is a second trade-off: performance $v s$. cost (or scalability) (Fig. 15b). New materials often possess extraordinary performances (i.e., high permeability and selectivity), but they suffer from limited scalability, high cost and low compatibility with traditional materials. One potential solution to this dilemma is to reversely engineer conventional and scalable materials (e.g., polymers) to mimic, or partly mimic the structures of these novel materials (e.g., biological channels or synthetically porous and selective materials). We need to strike a balance between performance and cost. The engineered separation materials can approach (or partially approach) the performances of novel materials and maintain the original scalability. The enhancement may not be as remarkable as those novel materials. However, as long as the technology is transformative and scalable, the implementation of the new technologies can result in significant reduction in both capital and operational costs, even providing $10-20 \%$ selectivity enhancement. In this regard, the bioinspired concept can be thought as successful in industrial scale applications. We may see this idea from several recent studies on polyamide RO

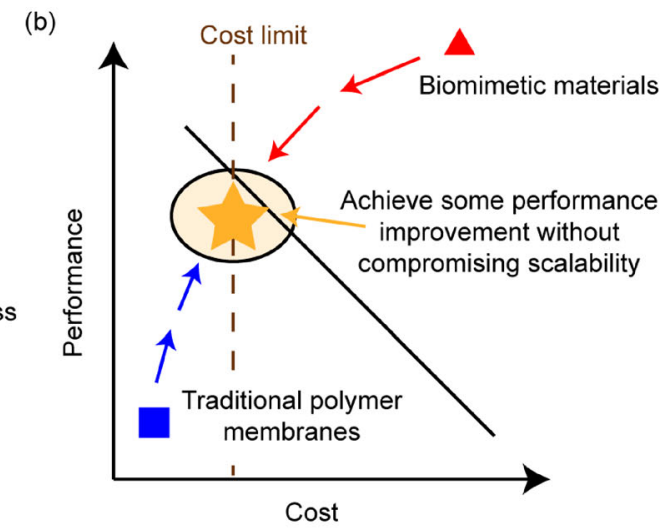

Fig. 15 Challenges for bioinspired and biomimetic membranes. (a) The Gartner Hype Cycle. Reproduced from https://en.wikipedia.org/ wiki/Hype_cycle. Most newly invented technologies follow this trend, including the majority of the novel materials developed in academia for separations. (b) Performance-cost trade-off for separation materials. A cost limit should be set for a new technology or material. Within that range, we should make efforts to improve separation efficiency by either reengineering traditional materials or downgrading biomimetic materials to achieve certain scalability. 
membranes that employed a couple of novel technologies (e.g., templated interfacial polymerization, additive manufacturing and 3D printing) (Chowdhury et al., 2018a; Tan et al., 2018; Wang et al., 2018; Liang et al., 2020). The resulting 'new' RO membranes showed unique internal nanoscale structures and enhanced transport properties. Recently, one of the artificial water channels discussed in the paper, imidazole I-quartets from Barboiu group, has been integrated into polyamide film of RO membrane during the interfacial polymerization process and the resulting membrane showed $99.5 \%$ rejection of $\mathrm{NaCl}$ or $91.4 \%$ rejection of boron with a water permeability of $\sim 2.5$ LMH/bar (Di Vincenzo et al., 2020). This doping approach could be scalable if the process is optimized and the assynthesized membranes are defect-free and exhibit the embedded channels' functionality.

The third key for bioinspired and biomimetic membranes is even if these novel materials cannot be largely scaled up, they can be optimized and targeted at special applications with highly rewarding products. In addition to BWCs, a plenty of biological channels are good candidates to mimic (e.g., ion-selective channels (Carmichael et al., 1989; Voyer and Robitaille, 1995; Dhakshnamoorthy et al., 2016; Kumar et al., 2016; Sakipov et al., 2018) and chiral channels (Chen et al., 2013; Klaerke et al., 2018; Kocsis et al., 2018a) that can be used in pharmaceutical, metal refinery and battery industries. Since treatment capacity in these fields may not be as large as that in water and wastewater treatment and the feed stream is often well pretreated with little contaminants, bioinspired and biomimetic membranes with small or moderate scales could handle the process and achieve much better separation efficiency.

Open Access This article is licensed under a Creative Commons Attribution 4.0 International License, which permits use, sharing, adaptation, distribution and reproduction in any medium or format, as long as you give appropriate credit to the original author(s) and the source, provide a link to the Creative Commons licence, and indicate if changes were made. The images or other third party material in this article are included in the article's Creative Commons licence, unless indicated otherwise in a credit line to the material. If material is not included in the article's Creative Commons licence and your intended use is not permitted by statutory regulation or exceeds the permitted use, you will need to obtain permission directly from the copyright holder. To view a copy of this licence, visit http://creativecommons.org/licenses/by/4.0/.

\section{Abbreviation}

Aquaporins: AQPs

Aquaporin 0: AQP0

Aquaporin 1: AQP1

Aquaporin Z: AqpZ

Aquaporin from Rhodobacter sphaeroides: RsAqpZ

Artificial water channels: AWCs

$\alpha$-Hemolysin: $\alpha \mathrm{HL}$
Biological water channels: BWCs

4,4'-Bis(4'-(N,N-bis(6"-(N,N,N-trimethylammonium)hexyl)amino)-styryl) stilbene tetraiodide: DSSN +

Block copolymers: BCPs

Carbon nanotubes: CNTs

Carbon nanotube porins: CNTPs

Cellulose acetate: CA

Cork-screw design: CSD

Covalent organic frameworks: COFs

2-Dimentional: 2D

Diblock copolymers: di-BCPs

Ferric hydroxamate uptake protein component A: FhuA

Fluorescence correlation spectroscopy: FCS

Forward osmosis: FO

Lithium bis(trifluoromethanesulfonyl)imide: LiTFSI

$\mathrm{L} \cdot \mathrm{m}^{-2} \cdot \mathrm{h}^{-1}: \mathrm{LMH}$

Membrane proteins: MPs

Metal organic frameworks: MOFs

Molecular dynamics: MD

Molecular weight: MW

Molecular weight cut-off: MWCO

$m$-Phenylenediamine: MPD

Nanofiltration: NF

Off-center pore closure design: OCD

Octyl- $\beta, D$-glucoside: OG

Outer membrane protein F: OmpF

1-Palmitoyl-2-oleoyl-sn-glycero-3-phosphocholine: POPC

Peptide-appended hybrid[4]arene: PAH[4]

Peptide-appended pillar[5]arenes: PAP[5]

Phosphatidylcholine: PC

Phosphatidylserine: PS

Polyacrylonitrile: PAN

Poly(butadiene)- $b$-poly(ethylene oxide): PB-PEO

polycarbonate track etched membrane: PCTE

Poly(ethylenimine): PEI

Poly(3,4-ethylenedioxythiophene) polystyrene sulfonate: PEDOT:PSS

Poly(2-methyl-2-oxazoline)- $b$-poly-(dimethysiloxane)- $b$-poly(2-methyl-2oxazoline): PMOXA-

PDMS-PMOXA, ABA

Poly(isoprene)- $b$-poly(ethylene oxide)- $b$-poly(isoprene): IOI

Reverse osmosis: RO

Root-mean-squared deviation: RMSD

Thin film composite: TFC

Transmission electron microscopy: TEM

Triblock copolymers: tri-BCPs

Ultraviolet-visible: UV/vis

Uniform pore closure design: UCD 


\section{References}

Abdulsalam Ebrahim M, Karan S, Livingston A G (2020). On the influence of salt concentration on the transport properties of reverse osmosis membranes in high pressure and high recovery desalination. Journal of Membrane Science, 594: 117339

Adewole J K, Ahmad A L, Ismail S, Leo C P (2013). Current challenges in membrane separation of $\mathrm{CO}_{2}$ from natural gas: A review. International Journal of Greenhouse Gas Control, 17: 46-65

Agre P (2004). Aquaporin water channels (nobel lecture). Angewandte Chemie International Edition, 43(33): 4278-4290

Agre P, King L S, Yasui M, Guggino W B, Ottersen O P, Fujiyoshi Y, Engel A, Nielsen S (2002). Aquaporin water channels: From atomic structure to clinical medicine. Journal of Physiology, 542(1): 3-16

Aksimentiev A, Schulten K (2005). Imaging $\alpha$-hemolysin with molecular dynamics: Ionic conductance, osmotic permeability, and the electrostatic potential map. Biophysical Journal, 88(6): 37453761

Aquaporin A/S.Available online at https://aquaporin.com/

Balaram V (2019). Rare earth elements: A review of applications, occurrence, exploration, analysis, recycling, and environmental impact. Geoscience Frontiers, 10(4): 1285-1303

Barakat M A (2011). New trends in removing heavy metals from industrial wastewater. Arabian Journal of Chemistry, 4(4): 361-377

Barboiu M (2012). Artificial water channels. Angewandte Chemie International Edition, 51(47): 11674-11676

Barboiu M (2016). Artificial water channels: Incipient innovative developments. Chemical Communications, 52(33): 5657-5665

Barboiu M, Gilles A (2013). From natural to bioassisted and biomimetic artificial water channel system. Accounts of Chemical Research, 46(12): 2814-2823

Belegrinou S, Dorn J, Kreiter M, Kita-Tokarczyk K, Sinner E K, Meier W (2010). Biomimetic supported membranes from amphiphilic block copolymers. Soft Matter, 6(1): 179-186

Belluati A, Mikhalevich V, Yorulmaz Avsar S, Daubian D, Craciun I, Chami M, Meier W P, Palivan C G (2020). How do the properties of amphiphilic polymer membranes influence the functional insertion of peptide pores? Biomacromolecules, 21(2): 701-715

Benrabah D, Baril D, Sanchez J Y, Armand M, Heres B P S, Gard G G (1993). Comparative electrochemical study of new poly(oxyethylene)-Li salt complexes. Journal of the Chemical Society, Faraday Transactions, 89(2): 355-359

Böckmann R A, De Groot B L, Kakorin S, Neumann E, Grubmüller H (2008). Kinetics, statistics, and energetics of lipid membrane electroporation studied by molecular dynamics simulations. Biophysical Journal, 95(4): 1837-1850

Borgnia M, Nielsen S, Engel A, Agre P (1999b). Cellular and molecular biology of the aquaporin water channels. Annual Review of Biochemistry, 68(1): 425-458

Borgnia M J, Kozono D, Calamita G, Maloney P C, Agre P, Ambientale G (1999a). Functional reconstitution and characterization of AqpZ, the E . coli water channel protein. 291(5): 1169-1179

Bornhorst J, Falke J J (2010). Purification of proteins using polyhistidine affinity tags. Methods in Enzymology, 2000(326): 245-254

Branton D, Deamer D W, Marziali A, Bayley H, Benner S A, Butler T, Di Ventra M, Garaj S, Hibbs A, Huang X, Jovanovich S B, Krstic P S,
Lindsay S, Ling X S, Mastrangelo C H, Meller A, Oliver J S, Pershin Y V, Ramsey J M, Riehn R, Soni G V, Tabard-Cossa V, Wanunu M, Wiggin M, Schloss J A (2008). The potential and challenges of nanopore sequencing. Nature Biotechnology, 26(10): 1146-1153

Burger B, Maffettone P M, Gusev V V, Aitchison C M, Bai Y, Wang X, Li X, Alston B M, Li B, Clowes R, Rankin N, Harris B, Sprick R S, Cooper A I (2020). A mobile robotic chemist. Nature, 583(7815): 237-241

Calamita G, Bishai W R, Preston G M, Guggino W B, Agre P (1995). Molecular cloning and characterization of AqpZ, a water channel from Escherichia coli. Journal of Biological Chemistry, 270(49): 29063-29066

Carmichael V E, Dutton P J, Fyles T M, James T D, Swan J A, Zojaji M (1989). Biomimetic ion transport: A functional model of a unimolecular ion channel. Journal of the American Chemical Society, 111(2): 767-769

Cheisson T, Schelter E J (2019). Rare earth elements: Mendeleev's bane, modern marvels. Science, 363(6426): 489-493

Chen L, Si W, Zhang L, Tang G, Li Z T, Hou J L (2013). Chiral selective transmembrane transport of amino acids through artificial channels. Journal of the American Chemical Society, 135(6): 2152-2155

Chen X, Zhang H, Tunuguntla R H, Noy A (2019). Silicon nanoribbon $\mathrm{pH}$ sensors protected by a barrier membrane with carbon nanotube porins. Nano Letters, 19(2): 629-634

Chowdhury M R, Steffes J, Huey B D, McCutcheon J R (2018a). 3D printed polyamide membranes for desalination. Science, 361(6403): 682-686

Chowdhury R, Ren T, Shankla M, Decker K, Grisewood M, Prabhakar J, Baker C, Golbeck J H, Aksimentiev A, Kumar M, Maranas C D (2018b). PoreDesigner for tuning solute selectivity in a robust and highly permeable outer membrane pore. Nature Communications, 9(1): 3661

Chrispeels M J, Agre P (1994). Aquaporins: water channel proteins of plant and animal cells. Trends in Biochemical Sciences, 19(10): 421425

Chun Y, Qing L, Sun G, Bilad M R, Fane A G, Chong T H (2018). Prototype aquaporin-based forward osmosis membrane: Filtration properties and fouling resistance. Desalination, 445: 75-84

Cohen S M (2012). Postsynthetic methods for the functionalization of metal-organic frameworks. Chemical Reviews, 112(2): 970-1000

Compton O C, Nguyen S T (2010). Graphene oxide, highly reduced graphene oxide, and graphene: Versatile building blocks for carbonbased materials. Small, 6(6): 711-723

Connolly D L, Shanahan C M, Weissberg P L (1998). The aquaporins. A family of water channel proteins. International Journal of Biochemistry \& Cell Biology, 30(2): 169-172

Cragg P J, Sharma K (2012). Pillar[5]arenes: Fascinating cyclophanes with a bright future. Chemical Society Reviews, 41(2): 597-607

Dalane K, Dai Z, Mogseth G, Hillestad M, Deng L (2017). Potential applications of membrane separation for subsea natural gas processing: A review. Journal of Natural Gas Science and Engineering, 39: 101-117

Dhakshnamoorthy B, Rohaim A, Rui H, Blachowicz L, Roux B (2016). Structural and functional characterization of a calcium-activated cation channel from Tsukamurella paurometabola. Nature Communications, 7(1): 12753 
Di Vincenzo M, Tiraferri A, Musteata V, Chisca S, Sougrat R, Huang L (2020). Biomimetic artificial water channel membranes for enhanced desalination. Nature Nanotechnology, https://doi.org/10.1038/ s41565-020-00796-x

Dorn J, Belegrinou S, Kreiter M, Sinner E K, Meier W (2011). Planar block copolymer membranes by vesicle spreading. Macromolecular Bioscience, 11(4): 514-525

Duong P H H, Chung T S, Jeyaseelan K, Armugam A, Chen Z, Yang J, Hong M (2012). Planar biomimetic aquaporin-incorporated triblock copolymer membranes on porous alumina supports for nanofiltration. Journal of Membrane Science, 409-410: 34-43

Elimelech M, Phillip W A (2011). The future of seawater desalination: Energy, technology, and the environment. Science, 333(6043): 712717

Epsztein R, DuChanois R M, Ritt C L, Noy A, Elimelech M (2020). Towards single-species selectivity of membranes with subnanometre pores. Nature Nanotechnology, 15(6): 426-436

Erbakan M, Shen Y X, Grzelakowski M, Butler P J, Kumar M, Curtis W R (2014). Molecular cloning, overexpression and characterization of a novel water channel protein from Rhodobacter sphaeroides. PLoS One, 9(1): e86830

Ersson B, Rydén L, Janson J C (2011). In: Janson J C, eds. Protein purification: Principles, high resolution methods, and applications. 3rd ed. Hoboken: Wiley

Falagán C, Grail B M, Johnson D B (2017). New approaches for extracting and recovering metals from mine tailings. Minerals Engineering, 106: 71-78

Fei Z, Zhao D, Geldbach T J, Scopelliti R, Dyson P J, Antonijevic S, Bodenhausen $G$ (2005). A synthetic zwitterionic water channel: Characterization in the solid state by X-ray crystallography and NMR spectroscopy. Angewandte Chemie International Edition, 44(35): $5720-5725$

Feng H, Lu X, Wang W, Kang N G, Mays J W (2017). Block copolymers: Synthesis, self-assembly, and applications. Polymers, 9 (10): 494

Flory P J, Krigbaum W R (1951). Thermodynamics of high polymer solutions. Annual Review of Physical Chemistry, 2(1): 383-402

Freeman B D (1999). Basis of permeability/selectivity tradeoff relations in polymeric gas separation membranes. Macromolecules, 32(2): 375-380

Fujiyoshi Y (1998). The structural study of membrane proteins by electron crystallography. Advances in Biophysics, 35: 25-80

Fuwad A, Ryu H, Malmstadt N, Kim S M, Jeon T J (2019). Biomimetic membranes as potential tools for water purification: Preceding and future avenues. Desalination, 458: 97-115

Fyles T M (2007). Synthetic ion channels in bilayer membranes. Chemical Society Reviews, 36(2): 335-347

Garner L E, Park J, Dyar S M, Chworos A, Sumner J J, Bazan G C (2010). Modification of the optoelectronic properties of membranes via insertion of amphiphilic phenylenevinylene oligoelectrolytes. Journal of the American Chemical Society, 132(29): 10042-10052

Geng J, Kim K, Zhang J, Escalada A, Tunuguntla R, Comolli L R, Allen F I, Shnyrova A V, Cho K R, Munoz D, Wang Y M, Grigoropoulos C P, Ajo-Franklin C M, Frolov V A, Noy A (2014). Stochastic transport through carbon nanotubes in lipid bilayers and live cell membranes. Nature, 514(7524): 612-615
Gin D L, Noble R D, (2011). Designing the next generation of chemical separation membranes. Science, 332(6030): 674-676

Giwa A, Hasan S W, Yousuf A, Chakraborty S, Johnson D J, Hilal N (2017). Biomimetic membranes: A critical review of recent progress. Desalination, 420: 403-424

Gomes D, Agasse A, Thiébaud P, Delrot S, Gerós H, Chaumont F (2009). Aquaporins are multifunctional water and solute transporters highly divergent in living organisms. Biochimica et Biophysica ActaBiomembranes, 1788(6): 1213-1228

Gonen T, Sliz P, Kistler J, Cheng Y, Walz T (2004). Aquaporin-0 membrane junctions reveal the structure of a closed water pore. Nature, 429(6988): 193-197

Gonen T, Walz T (2006). The structure of aquaporins. Quarterly Reviews of Biophysics, 39(4): 361-396

Górecki R, Reurink D M, Khan M M, Sanahuja-Embuena V, Trzaskuś K, Hélix-Nielsen C (2020). Improved reverse osmosis thin film composite biomimetic membranes by incorporation of polymersomes. Journal of Membrane Science, 593: 117392

Greenlee L F, Lawler D F, Freeman B D, Marrot B, Moulin P (2009). Reverse osmosis desalination: Water sources, technology, and today's challenges. Water Research, 43(9): 2317-2348

Grzelakowski M, Cherenet M F, Shen Y X, Kumar M (2015). A framework for accurate evaluation of the promise of aquaporin based biomimetic membranes. Journal of Membrane Science, 479: 223231

Guo S, Dong S (2011). Graphene nanosheet: Synthesis, molecular engineering, thin film, hybrids, and energy and analytical applications. Chemical Society Reviews, 40(5): 2644-2672

Guo W, Ngo H H, Li J (2012). A mini-review on membrane fouling. Bioresource Technology, 122: 27-34

Habel J, Hansen M, Kynde S, Larsen N, Midtgaard S R, Jensen G V, Bomholt J, Ogbonna A, Almdal K, Schulz A, Hélix-Nielsen C (2015). Aquaporin-based biomimetic polymeric membranes: Approaches and challenges. Membranes, 5(3): 307-351

Hancock R E W, Carey A M (1979). Outer membrane of Pseudomonas aeruginosa: Heat- and 2-mercaptoethanol-modifiable proteins. Journal of Bacteriology, 140(3): 902-910

Harrison R G, Todd P, Rudge S R, Petrides D P (2015). In: Harrison R G, eds. Bioseparations Science and Engineering. 1st ed. New York: Oxford University Press

Hasell T, Cooper A I (2016). Porous organic cages: Soluble, modular and molecular pores. Nature Reviews. Materials, 1(9): 16053

Hasler L, Heymann J B, Engel A, Kistler J, Walz T (1998). 2D crystallization of membrane proteins: Rationales and examples. Journal of Structural Biology, 121(2): 162-171

Hélix-Nielsen C (2009). Biomimetic membranes for sensor and separation applications. Analytical and Bioanalytical Chemistry, 395(3): 697-718

Hélix-Nielsen C (2018). Biomimetic membranes as a technology platform: Challenges and opportunities. Membranes, 8(3): 44

Hinds B J, Chopra N, Rantell T, Andrews R, Gavalas V, Bachas L G (2004). Aligned multiwalled carbon nanotube membranes. Science, 303(5654): 62-65

Hodnik N, Baldizzone C, Polymeros G, Geiger S, Grote J P, Cherevko S, Mingers A, Zeradjanin A, Mayrhofer K J J (2016). Platinum recycling going green via induced surface potential alteration 
enabling fast and efficient dissolution. Nature Communications, 7(1): 13164

Holme J P, Hansen J S, Vissing T, Perry M. E, Hélix-Nielsen C (2015). Biomimetic membranes and uses thereof. US20150360183A1

Holt J K, Park H G, Wang Y, Stadermann M, Artyukhin A B, Grigoropoulos C P (2006). Fast mass transport through sub-2nanometer carbon nanotubes. Science, 312(5776): 1034-1037

Hong H, Tamm L K (2004). Elastic coupling of integral membrane protein stability to lipid bilayer forces. Proceedings of the National Academy of Sciences of the United States of America, 101(12): 4065-4070

Hoomann T, Jahnke N, Horner A, Keller S, Pohl P (2013). Filter gate closure inhibits ion but not water transport through potassium channels. Proceedings of the National Academy of Sciences of the United States of America, 110(26): 10842-10847

Horner A, Pohl P (2018). Single-file transport of water through membrane channels. Faraday Discussions, 209: 9-33

Horner A, Zocher F, Preiner J, Ollinger N, Siligan C, Akimov S A, Pohl $P$ (2015). The mobility of single-file water molecules is governed by the number of $\mathrm{H}$-bonds they may form with channel-lining residues. Science Advances, 1(2): e1400083

Hovijitra N T, Wuu J J, Peaker B, Swartz J R (2009). Cell-free synthesis of functional aquaporin $\mathrm{Z}$ in synthetic liposomes. Biotechnology and Bioengineering, 104(1): 40-49

Hu X B, Chen Z, Tang G, Hou J L, Li Z T (2012). Single-molecular artificial transmembrane water channels. Journal of the American Chemical Society, 134(20): 8384-8387

Hub J S, Grubmüller H, de Groot B L (2009). In: Beitz E, ed. Dynamics and energetics of permeation through aquaporins. What do we learn from molecular dynamics simulations? BT-Aquaporins. Berlin: Springer, $57-76$

Huggins M L (1942). Some properties of solutions of long-chain compounds. Journal of Physical Chemistry, 46(1): 151-158

Humphrey W, Dalke A, Schulten K (1996). VMD: Visual Molecular Dynamics. Journal of Molecular Graphics, 14(1): 33-38

Huo Y, Zeng H (2016). "Sticky"-Ends-Guided creation of functional hollow nanopores for guest encapsulation and water transport. Accounts of Chemical Research, 49(5): 922-930

Israelachvili J N, Mitchell D J, Ninham B W (1977). Theory of selfassembly of lipid bilayers and vesicles. BBA- Biomembranes, 470(2): 185-201

Jap B K, Walian P J, Gehring K (1991). Structural architecture of an outer membrane channel as determined by electron crystallography. Nature, 350(6314): 167-170

Jörg V, Groth Jesper S, Hoier N K, Oliver G (2015). Membranes, Hollow fiber module having tfc-aquaporin modified. US2015144553A1

Kalaj M, Bentz K C, Ayala S Jr, Palomba J M, Barcus K S, Katayama Y, Cohen S M (2020). MOF-Polymer Hybrid Materials: From simple composites to tailored architectures. Chemical Reviews, 120(16): $8267-8302$

Kaler E W, Murthy A K, Rodriguez B E, Zasadzinski J A N (1989). Spontaneous vesicle formation in aqueous mixtures of single-tailed surfactants. Science, 245(4924): 1371-1374

Kalé L, Skeel R, Bhandarkar M, Brunner R, Gursoy A, Krawetz N, Phillips J, Shinozaki A, Varadarajan K, Schulten K. (1999). NAMD2:
Greater scalability for parallel molecular dynamics. Journal of Computational Physics, 151(1): 283-312

Kaucher M S, Peterca M, Dulcey A E, Kim A J, Vinogradov S A, Hammer D A, Heiney P A, Percec V (2007). Selective transport of water mediated by porous dendritic dipeptides. Journal of the American Chemical Society, 129(38): 11698-11699

Kaufman Y, Berman A, Freger V (2010). Supported lipid bilayer membranes for water purification by reverse osmosis. Langmuir, 26(10): 7388-7395

Kaufman Y, Grinberg S, Linder C, Heldman E, Gilron J, Shen Y X, Kumar M, Lammertink R G H, Freger V (2014). Towards supported bolaamphiphile membranes for water filtration: Roles of lipid and substrate. Journal of Membrane Science, 457: 50-61

Kita-Tokarczyk K, Grumelard J, Haefele T, Meier W (2005). Block copolymer vesicles: Using concepts from polymer chemistry to mimic biomembranes. Polymer, 46(11): 3540-3563

Klaerke D A, Tejada M L A, Christensen V G, Lassen M, Pedersen P A, Calloe K (2018). Reconstitution and electrophysiological characterization of ion channels in lipid bilayers. Current Protocols in Pharmacology, 81(1): e37

Klara S S, Saboe P O, Sines I T, Babaei M, Chiu P L, Dezorzi R, Dayal K, Walz T, Kumar M, Mauter M S (2016). Magnetically directed two-dimensional crystallization of OmpF membrane proteins in block copolymers. Journal of the American Chemical Society, 138(1): 28-31

Kocsis I, Sorci M, Vanselous H, Murail S, Sanders S E, Licsandru E (2018a). Oriented chiral water wires in artificial transmembrane channels. Science Advances, 4(3): eaao5603

Kocsis I, Sun Z, Legrand Y M, Barboiu M (2018b). Artificial water channels - deconvolution of natural aquaporins through synthetic design. NPJ Clean Water, 1(1): 13

Köper I (2007). Insulating tethered bilayer lipid membranes to study membrane proteins. Molecular BioSystems, 3(10): 651-657

Koros W J, Zhang C (2017). Materials for next-generation molecularly selective synthetic membranes. Nature Materials, 16(3): 289-297

Kruse E, Uehlein N, Kaldenhoff R (2006). The aquaporins. Genome Biology, 7(2): 206

Kumar M, Grzelakowski M, Zilles J, Clark M, Meier W (2007). Highly permeable polymeric membranes based on the incorporation of the functional water channel protein Aquaporin Z. Proceedings of the National Academy of Sciences of the United States of America, 104(52): 20719-20724

Kumar M, Habel J E O, Shen Y X, Meier W P, Walz T (2012). Highdensity reconstitution of functional water channels into vesicular and planar block copolymer membranes. Journal of the American Chemical Society, 134(45): 18631-18637

Kumar M, Shen Y X, Saboe P O (2013). Biological and biomimetic membranes. In: Hoek E M V, ed. Encyclopedia of Membrane Science and Technology. 1st ed. Hoboken: Wiley, 1-37

Kumar Y P, Das R N, Schütte O M, Steinem C, Dash J (2016). Bistriazolyl diguanosine derivatives as synthetic transmembrane ion channels. Nature Protocols, 11(6): 1039-1056

Kutzner C, Grubmüller H, De Groot B L, Zachariae U (2011). Computational electrophysiology: The molecular dynamics of ion channel permeation and selectivity in atomistic detail. Biophysical Journal, 101(4): 809-817 
Lang C, Shen Y X, LaNasa J A, Ye D, Song W, Zimudzi T J, Hickner M A, Gomez E D, Gomez E W, Kumar M, Hickey R J (2018). Creating cross-linked lamellar block copolymer supporting layers for biomimetic membranes. Faraday Discussions, 209: 179-191

Lang C, Ye D, Song W, Yao C, Tu Y M, Capparelli C, LaNasa J A, Hickner M A, Gomez E W, Gomez E D, Hickey R J, Kumar M (2019). Biomimetic separation of transport and matrix functions in lamellar block copolymer channel-based membranes. ACS Nano, 13(7): 8292-8302

Latimer P, Pyle B E (1972). Light scattering at various angles. Biophysical Journal, 12(7): 764-773

Le Duc Y, Michau M, Gilles A, Gence V, Legrand Y M, Vanderlee A, Tingry S, Barboiu M (2011). Imidazole-quartet water and proton dipolar channels. Angewandte Chemie International Edition, 50(48): 11366-11372

Lehn B J (1990). Perspectives in supramolecular chemistry-from molecular recognition towards molecular information processing and self-organization. Angewandte Chemie International Edition, 29(11): 1304-1319

Lehn J M (1988). Supramolecular chemistry-scope and perspectives molecules, supermolecules, and molecular devices. Angewandte Chemie International Edition, 27(1): 89-112

Lei J C, Zhang X, Zhou Z (2015). Recent advances in MXene: Preparation, properties, and applications. Frontiers in Physics, 10(3): 276-286

Li M, Xiong Y, Qing G (2020). Smart bio-separation materials. Trends in Analytical Chemistry, 124: 115585

Li Q, Li X, Ning L, Tan C H, Mu Y, Wang R (2019a). Hyperfast water transport through biomimetic nanochannels from peptide-attached (pR)-pillar[5]arene. Small, 15(6): 1804678

Li X, Chou S, Wang R, Shi L, Fang W, Chaitra G, Tang C Y, Torres J, $\mathrm{Hu} \mathrm{X}$, Fane A G (2015). Nature gives the best solution for desalination: Aquaporin-based hollow fiber composite membrane with superior performance. Journal of Membrane Science, 494: 6877

Li X, Loh C H, Wang R, Widjajanti W, Torres J (2017a). Fabrication of a robust high-performance FO membrane by optimizing substrate structure and incorporating aquaporin into selective layer. Journal of Membrane Science, 525: 257-268

Li X, Wang R, Tang C, Vararattanavech A, Zhao Y, Torres J, Fane T (2012). Preparation of supported lipid membranes for aquaporin $Z$ incorporation. Colloids and Surfaces. B, Biointerfaces, 94: 333-340

Li X, Wang R, Wicaksana F, Tang C, Torres J, Fane A G (2014). Preparation of high performance nanofiltration (NF) membranes incorporated with aquaporin Z. Journal of Membrane Science, 450: 181-188

Li Y, Qi S, Tian M, Widjajanti W, Wang R (2019b). Fabrication of aquaporin-based biomimetic membrane for seawater desalination. Desalination, 467: 103-112

Li Z, Valladares Linares R, Bucs S, Fortunato L, Hélix-Nielsen C, Vrouwenvelder J S, Ghaffour N, Leiknes T O, Amy G (2017b). Aquaporin based biomimetic membrane in forward osmosis: Chemical cleaning resistance and practical operation. Desalination, 420: 208-215

Liang Y, Zhu Y, Liu C, Lee K R, Hung W S, Wang Z, Li Y, Elimelech $\mathrm{M}$, Jin J, Lin S (2020). Polyamide nanofiltration membrane with highly uniform sub-nanometre pores for sub-1 $\AA$ precision separation. Nature Communications, 11(1): 2015

Licsandru E, Kocsis I, Shen Y X, Murail S, Legrand Y M, Van Der Lee A, Tsai D, Baaden M, Kumar M, Barboiu M (2016). Salt-excluding artificial water channels exhibiting enhanced dipolar water and proton translocation. Journal of the American Chemical Society, 138(16): 5403-5409

Liu G, Jin W, Xu N (2016). Two-dimensional-material membranes: A new family of high-performance separation membranes. Angewandte Chemie International Edition, 55(43): 13384-13397

Liu G, Zhao Z, Ghahreman A (2019a). Novel approaches for lithium extraction from salt-lake brines: A review. Hydrometallurgy, 187: 81-100

Liu K, Tian Y, Jiang L (2013). Bio-inspired superoleophobic and smart materials: Design, fabrication, and application. Progress in Materials Science, 58(4): 503-564

Liu M, Wang S, Jiang L (2017). Nature-inspired superwettability systems. Nature Reviews. Materials, 2(7): 17036

Liu M, Zhang L, Little M A, Kapil V, Ceriotti M, Yang S, Ding L, Holden D L, Balderas-Xicohténcatl R, He D, Clowes R, Chong S Y, Schütz G, Chen L, Hirscher M, Cooper A I (2019b). Barely porous organic cages for hydrogen isotope separation. Science, 366(6465): 613-620

Luo W, Xie M, Song X, Guo W, Ngo H H, Zhou J L, Nghiem L D (2018). Biomimetic aquaporin membranes for osmotic membrane bioreactors: Membrane performance and contaminant removal. Bioresource Technology, 249: 62-68

MacKerell A D Jr, Bashford D, Bellott M, Dunbrack R L Jr, Evanseck J D, Field M J, Fischer S, Gao J, Guo H, Ha S, Joseph-McCarthy D, Kuchnir L, Kuczera K, Lau F T K, Mattos C, Michnick S, Ngo T, Nguyen D T, Prodhom B, Reiher W E, Roux B, Schlenkrich M, Smith J C, Stote R, Straub J, Watanabe M, Wiórkiewicz-Kuczera J, Yin D, Karplus M (1998). All-atom empirical potential for molecular modeling and dynamics studies of proteins. Journal of Physical Chemistry B, 102(18): 3586-3616

Madsen H T, Bajraktari N, Hélix-Nielsen C, Van der Bruggen B, Søgaard E G (2015). Use of biomimetic forward osmosis membrane for trace organics removal. Journal of Membrane Science, 476: 469474

Mai Y, Eisenberg A (2012). Self-assembly of block copolymers. Chemical Society Reviews, 41(18): 5969-5985

Malinova V, Belegrinou S, de Bruyn Ouboter D, Meier W P (2010). In: Meier W P, Knoll W, eds. Biomimetic Block Copolymer Membranes. Berlin: Springer, 87-111

Masi M, Pagès J M (2013). Structure, function and regulation of outer membrane proteins involved in drugt transport in enterobactericeae: the $\mathrm{OmpF} / \mathrm{C}-\mathrm{TolC}$ Case. Open Microbiology Journal, 7(1): 22-33

Matile S, Vargas Jentzsch A, Montenegro J, Fin A (2011). Recent synthetic transport systems. Chemical Society Reviews, 40(5): 24532474

McCutcheon J R (2019). Avoiding the hype in developing commercially viable desalination Technologies. Joule, 3(5): 1168-1171

Meinild A K, Klaerke D A, Zeuthen T (1998). Bidirectional water fluxes and specificity for small hydrophilic molecules in aquaporins $0-5$. Journal of Biological Chemistry, 273(49): 32446-32451

Mentzel S, Perry M E, Vogel J, Braekevelt S, Geschke O, Larsen M E S 
(2014). Systems for water extraction. WO2014128293Al

Miao Y, Johnson N W, Phan T, Heck K, Gedalanga P B, Zheng X, Adamson D, Newell C, Wong M S, Mahendra S (2020). Monitoring, assessment, and prediction of microbial shifts in coupled catalysis and biodegradation of 1,4-dioxane and co-contaminants. Water Research, 173: 115540

Mohammad M M, Howard K R, Movileanu L (2011). Redesign of a plugged $\beta$-barrel membrane protein. Journal of Biological Chemistry, 286(10): 8000-8013

Murata K, Mitsuoka K, Hirai T, Walz T, Agre P, Heymann J B (2000). Structural determinants of water permeation through aquaporin-. Nature, 407(6804): 599-605

Nagai A, Guo Z, Feng X, Jin S, Chen X, Ding X, Jiang D (2011). Pore surface engineering in covalent organic frameworks. Nature Communications, 2(1): 536

Nassrullah H, Anis S F, Hashaikeh R, Hilal N (2020). Energy for desalination: A state-of-the-art review. Desalination, 491: 114569

Nath A, Atkins W M, Sligar S G (2007). Applications of phospholipid bilayer nanodiscs in the study of membranes and membrane proteins. Biochemistry, 46(8): 2059-2069

Nephrol S (1998). Decreased membrane hypercalcemic aquaporin-2 delivery rats expression in kidney and collecting apical ducts plasma of polyuric. Journal of the American Society of Nephrology, 9(2): 2181-2193

Ogoshi T, Kanai S, Fujinami S, Yamagishi T A, Nakamoto Y (2008). Para-bridged symmetrical pillar[5]arenes: Their Lewis acid catalyzed synthesis and host-guest property. Journal of the American Chemical Society, 130(15): 5022-5023

Ogoshi T, Yamagishi T A, Nakamoto Y (2016). Pillar-shaped macrocyclic hosts pillar[n]arenes: New key players for supramolecular chemistry. Chemical Reviews, 116(14): 7937-8002

Okamoto Y, Lienhard J H (2019). How RO membrane permeability and other performance factors affect process cost and energy use: A review. Desalination, 470: 114064

Park H B, Kamcev J, Robeson L M, Elimelech M, Freeman B D (2017). Maximizing the right stuff: The trade-off between membrane permeability and selectivity. Science, 356(6343): eaab0530

Peng B, Tang J, Luo J, Wang P, Ding B, Tam K C (2018). Applications of nanotechnology in oil and gas industry: Progress and perspective. Canadian Journal of Chemical Engineering, 96(1): 91-100

Plançon L, Chami M, Letellier L (1997). Reconstitution of FhuA, an Escherichia coli outer membrane protein, into liposomes: Binding of phage T5 to FhuA triggers the transfer of DNA into the proteoliposomes. Journal of Biological Chemistry, 272(27): 1686816872

Pollmann K, Kutschke S, Matys S, Kostudis S, Hopfe S, Raff J (2016). Novel biotechnological approaches for the recovery of metals from primary and secondary resources. Minerals (Basel), 6(2): 54

Porter C J, Werber J R, Zhong M, Wilson C J, Elimelech M (2020). Pathways and challenges for biomimetic desalination membranes with sub-nanometer channels. ACS Nano, 14(9): 10894-10916

Preston G M, Carroll T P, Guggino W B, Agre P (1992). Appearance of water channels in Xenopus oocytes expressing red cell CHIP28 protein. Science, 256(5055): 385-387

Qi S, Wang R, Chaitra G K M, Torres J, Hu X, Fane A G (2016). Aquaporin-based biomimetic reverse osmosis membranes: Stability and long term performance. Journal of Membrane Science, 508: 94103

Qiu S, Xue M, Zhu G (2014). Metal-organic framework membranes: From synthesis to separation application. Chemical Society Reviews, 43(16): 6116-6140

Rajesh S, Yan Y, Chang H C, Gao H, Phillip W A (2014). Mixed mosaic membranes prepared by layer-by-layer assembly for ionic separations. ACS Nano, 8(12): 12338-12345

Rathee V S, Qu S, Phillip W A, Whitmer J K (2016). A coarse-grained thermodynamic model for the predictive engineering of valenceselective membranes. Molecular Systems Design \& Engineering, 1(3): 301-312

Ren T, Erbakan M, Shen Y X, Barbieri E, Saboe P, Feroz H, Yan H, McCuskey S, Hall J F, Schantz A B, Bazan G C, Butler P J, Grzelakowski M, Kumar M (2017). Membrane protein insertion into and compatibility with biomimetic membranes. Advanced Biosystems, 1(7): 1700053

Rhoden V, Goldin S M (1979). Formation of unilamellar lipid vesicles of controllable dimensions by detergent dialysis. Biochemistry, 18(19): 4173-4176

Robeson L M (1991). Correlation of separation factor versus permeability for polymeric membranes. Journal of Membrane Science, 62(2): $165-185$

Robeson L M (2008). The upper bound revisited. Journal of Membrane Science, 320(1-2): 390-400

Saboe P O, Rapisarda C, Kaptan S, Hsiao Y S, Summers S R, de Zorzi R, Dukovski D, Yu J, de Groot B L, Kumar M, Walz T (2017). Role of pore-lining residues in defining the rate of water conduction by aquaporin-0. Biophysical Journal, 112(5): 953-965

Sabolic I, Valenti G, Verbavatz J M, Van Hoek A N, Verkman A S, Ausiello D A, Brown D (1992). Localization of the CHIP28 water channel in rat kidney. American Journal of Physiology. Cell Physiology, 263(6): C1225-C1233

Sakai N, Matile S (2013). Synthetic ion channels. Langmuir, 29(29): 9031-9040

Sakipov S, Sobolevsky A I, Kurnikova M G (2018). Ion permeation mechanism in epithelial calcium channel TRVP6. Scientific Reports, 8(1): 5715

Sanborn J R, Chen X, Yao Y, Hammons J A, Tunuguntla R H, Zhang Y, Newcomb C C, Soltis J A, de Yoreo J J, Van Buuren A, Parikh A N, Noy A (2018). Membranes: Carbon nanotube porins in amphiphilic block copolymers as fully synthetic mimics of biological membranes. Advanced Materials, 30(51): 1803355

Sanchez C, Arribart H, Giraud Guille M M(2005). Biomimetism and bioinspiration as tools for the design of innovative materials and systems. Nature Materials, 4(4): 277-288

Sanders C R II, Landis G C (1995). Reconstitution of membrane proteins into lipid-rich bilayered mixed micelles for NMR studies. Biochemistry, 34(12): 4030-4040

Sanders D F, Smith Z P, Guo R, Robeson L M, McGrath J E, Paul D R, Freeman B D (2013). Energy-efficient polymeric gas separation membranes for a sustainable future: A review. Polymer, 54(18): 4729-4761

Schneider S, Licsandru E D, Kocsis I, Gilles A, Dumitru F, Moulin E, Tan J, Lehn J M, Giuseppone N, Barboiu M (2017). Columnar selfassemblies of triarylamines as scaffolds for artificial biomimetic 
channels for ion and for water transport. Journal of the American Chemical Society, 139(10): 3721-3727

Scopes R K (1982). In: Scopes R K, eds. Protein purification: Principles and practice. 1st ed. Berlin: Springer

Seddon A M, Curnow P, Booth P J (2004). Membrane proteins, lipids and detergents: Not just a soap opera. Biochimica et Biophysica Acta- Biomembranes, 1666(1-2): 105-117

Shannon M A, Bohn P W, Elimelech M, Georgiadis J G, Mariñas B J, Mayes A M (2008). Science and technology for water purification in the coming decades. Nature, 452(7185): 301-310

Shen J, Fan J, Ye R, Li N, Mu Y, Zeng H (2020a). Polypyridine-based helical amide foldamer channels: Rapid transport of water and protons with high ion rejection. Angewandte Chemie International Edition, 59(32): 13328-13334

Shen J, Ye R, Romanies A, Roy A, Chen F, Ren C, Liu Z, Zeng H (2020b). Aquafoldmer-based aquaporin-like synthetic water channel. Journal of the American Chemical Society, 142(22): 10050-10058

Shen Y X, Saboe P O, Sines I T, Erbakan M, Kumar M (2014). Biomimetic membranes: A review. Journal of Membrane Science, 454: 359-381

Shen Y X, Si W, Erbakan M, Decker K, de Zorzi R, Saboe P O, Kang Y J, Majd S, Butler P J, Walz T, Aksimentiev A, Hou J, Kumar M (2015). Highly permeable artificial water channels that can selfassemble into two-dimensional arrays. Proceedings of the National Academy of Sciences of the United States of America, 112(32): 9810-9815

Shen Y X, Song W C, Barden D R, Ren T, Lang C, Feroz H (2018). Achieving high permeability and enhanced selectivity for Angstromscale separations using artificial water channel membranes. Nature Communications, 9(1): 2294

Shi B, Marchetti P, Peshev D, Zhang S, Livingston A G (2017). Will ultra-high permeance membranes lead to ultra-efficient processes? Challenges for molecular separations in liquid systems. Journal of Membrane Science, 525: 35-47

Sholl D S, Lively R P (2016). Seven chemical separations to change the world. Nature, 532(7600): 435-437

Si W, Xin P, Li Z T, Hou J L (2015). Tubular unimolecular transmembrane channels: Construction strategy and transport activities. Accounts of Chemical Research, 48(6): 1612-1619

Sianipar M, Kim S H, Khoiruddin K, Iskandar F, Wenten I G (2017). Functionalized carbon nanotube (CNT) membrane: Progress and challenges. RSC Advances, 7(81): 51175-51198

Sisson A L, Shah M R, Bhosale S, Matile S (2006). Synthetic ion channels and pores (2004-2005). Chemical Society Reviews, 35(12): 1269-1286

Song W, Joshi H, Chowdhury R, Najem J S, Shen Y X, Lang C, Henderson C B, Tu Y M, Farell M, Pitz M E, Maranas C D, Cremer P S, Hickey R J, Sarles S A, Hou J, Aksimentiev A, Kumar M (2020). Artificial water channels enable fast and selective water permeation through water-wire networks. Nature Nanotechnology, 15(1): 73-79

Song W, Kumar M (2019). Artificial water channels: toward and beyond desalination. Current Opinion in Chemical Engineering, 25: 9-17

Song W, Lang C, Shen Y, Kumar M (2018). Design considerations for artificial water channel-based membranes. Annual Review of Materials Research, 48(1): 57-82

Song W, Tu Y M, Oh H, Samineni L, Kumar M (2019). Hierarchical optimization of high-performance biomimetic and bioinspired membranes. Langmuir, 35(3): 589-607

Spulber M, Gerstandt K (2018). Diblock copolymer vesicles and separation membranes comprising aquaporin water channels and methods of making and using them. WO2018141985A1

Sullivan K, Zhang Y, Lopez J, Lowe M, Noy A (2020). Carbon nanotube porin diffusion in mixed composition supported lipid bilayers. Scientific Reports, 10(1): 11908

Sun G, Chung T S, Chen N, Lu X, Zhao Q (2013a). Highly permeable aquaporin-embedded biomimetic membranes featuring a magneticaided approach. RSC Advances, 3(24): 9178-9184

Sun G, Chung T S, Jeyaseelan K, Armugam A (2013b). A layer-by-layer self-assembly approach to developing an aquaporin-embedded mixed matrix membrane. RSC Advances, 3(2): 473-481

Sun G, Chung T S, Jeyaseelan K, Armugam A (2013c). Stabilization and immobilization of aquaporin reconstituted lipid vesicles for water purification. Colloids and Surfaces. B, Biointerfaces, 102: 466-471

Tabushi I, Kuroda Y, Yokota K (1982). A,B,D,F-tetrasubstituted $\beta$ cyclodextrin as artificial channel compound. Tetrahedron Letters, 23(44): 4601-4604

Tan Z, Chen S, Peng X, Zhang L, Gao C (2018). Polyamide membranes with nanoscale Turing structures for water purification. Science, 360(6388): 518-521

Tang C, Qiu C, Zhao Y, Shen W, Vararattanavech A, Wang R (2014). Aquaporin based thin film composite membranes. US2014332468

Tang C, Wang Z, Petrinić I, Fane A G, Hélix-Nielsen C (2015). Biomimetic aquaporin membranes coming of age. Desalination, 368 : 89-105

Tang C Y, Zhao Y, Wang R, Hélix-Nielsen C, Fane A G (2013). Desalination by biomimetic aquaporin membranes: Review of status and prospects. Desalination, 308: 34-40

Tu Y M, Song W, Ren T, Shen Y X, Chowdhury R, Rajapaksha P, Culp T E, Samineni L, Lang C, Thokkadam A, Carson D, Dai Y, Mukthar A, Zhang M, Parshin A, Sloand J N, Medina S H, Grzelakowski M, Bhattacharya D, Phillip W A, Gomez E D, Hickey R J, Wei Y, Kumar M (2020). Rapid fabrication of precise high-throughput filters from membrane protein nanosheets. Nature Materials, 19(3): 347-354

Tunuguntla R H, Allen F I, Kim K, Belliveau A, Noy A (2016a). Ultrafast proton transport in sub-1-nm diameter carbon nanotube porins. Nature Nanotechnology, 11(7): 639-644

Tunuguntla R H, Escalada A, Frolov V A, Noy A (2016b). Synthesis, lipid membrane incorporation, and ion permeability testing of carbon nanotube porins. Nature Protocols, 11(10): 2029-2047

Tunuguntla R H, Henley R Y, Yao Y C, Pham T A, Wanunu M, Noy A (2017). Enhanced water permeability and tunable ion selectivity in subnanometer carbon nanotube porins. Science, 357(6353): 792-796

Venkata Subbaiah Y P, Saji K J, Tiwari A (2016). Atomically Thin $\mathrm{MoS}_{2}$ : A Versatile nongraphene 2D material. Advanced Functional Materials, 26(13): 2046-2069

Verkman A S, Mitra A K (2000). Structure and function of aquaporin water channels. American Journal of Physiology. Renal Physiology, 278(1): F13-F28

Virkki L V, Cooper G J, Boron W F (2001). Cloning and functional expression of an MIP (AQP0) homolog from killifish (Fundulus heteroclitus) lens. American Journal of Physiology. Regulatory, Integrative and Comparative Physiology, 281(6): R1994-R2003 
Voyer N, Robitaille M (1995). A novel functional artificial ion channel. Journal of the American Chemical Society, 117(24): 6599-6600

Wagh P, Escobar I C (2019). Biomimetic and bioinspired membranes for water purification: A critical review and future directions. Environmental Progress \& Sustainable Energy, 38(3): e13215

Wagh P, Parungao G, Viola R E, Escobar I C (2015). A new technique to fabricate high-performance biologically inspired membranes for water treatment. Separation and Purification Technology, 156: 754765

Wagner S, Bader M L, Drew D, de Gier J W (2006). Rationalizing membrane protein overexpression. Trends in Biotechnology, 24(8): 364-371

Walz T, Hirai T, Murata K, Heymann J B, Mitsuoka K, Fujiyoshi Y, Smith B L, Agre P, Engel A (1997). The three-dimensional structure of aquaporin-1. Nature, 387(6633): 624-627

Wang H, Chung T S, Tong Y W, Jeyaseelan K, Armugam A, Chen Z, Hong M, Meier W (2012). Highly permeable and selective porespanning biomimetic membrane embedded with aquaporin $\mathrm{Z}$. Small, 8(8): 1185-1190

Wang H, Chung T S, Tong Y W, Meier W, Chen Z, Hong M, Jeyaseelan K, Armugam A (2011). Preparation and characterization of poresuspending biomimetic membranes embedded with Aquaporin $\mathrm{Z}$ on carboxylated polyethylene glycol polymer cushion. Soft Matter, 7(16): 7274-7280

Wang H L, Chung T S, Tong Y W, Jeyaseelan K, Armugam A, Duong H H P, Fu F, Seah H, Yang J, Hong M (2013). Mechanically robust and highly permeable AquaporinZ biomimetic membranes. Journal of Membrane Science, 434: 130-136

Wang M, Wang Z, Wang X, Wang S, Ding W, Gao C (2015). Layer-bylayer assembly of aquaporin z-incorporated biomimetic membranes for water purification. Environmental Science \& Technology, 49(6): 3761-3768

Wang Z, Wang Z, Lin S, Jin H, Gao S, Zhu Y, Jin J (2018). Nanoparticletemplated nanofiltration membranes for ultrahigh performance desalination. Nature Communications, 9(1): 2004

Wegst U G K, Bai H, Saiz E, Tomsia A P, Ritchie R O (2015). Bioinspired structural materials. Nature Materials, 14(1): 23-36

Werber J R, Deshmukh A, Elimelech M (2016a). The critical need for increased selectivity, not increased water permeability for desalination membranes. Environmental Science \& Technology Letters, 3(4): $112-120$

Werber J R, Elimelech M (2018). Permselectivity limits of biomimetic desalination membranes. Science Advances, 4(6): eaar8266

Werber J R, Osuji C O, Elimelech M (2016b). Materials for nextgeneration desalination and water purification membranes. Nature Reviews Materials, 1(5): 16018

Xia L, Andersen M F, Hélix-Nielsen C, McCutcheon J R (2017). Novel commercial aquaporin flat-sheet membrane for forward osmosis. Industrial \& Engineering Chemistry Research, 56(41): 1191911925

Xie M, Luo W, Guo H, Nghiem L D, Tang C Y, Gray S R (2018). Trace organic contaminant rejection by aquaporin forward osmosis membrane: Transport mechanisms and membrane stability. Water Research, 132: 90-98

Xie W, He F, Wang B, Chung T S, Jeyaseelan K, Armugam A, Tong Y W (2013). An aquaporin-based vesicle-embedded polymeric membrane for low energy water filtration. Journal of Materials Chemistry.

A, Materials for Energy and Sustainability, 1(26): 7592-7600

Yang Y, Walton A, Sheridan R, Güth K, Gauß R, Gutfleisch O, Buchert M, Steenari B M, Van Gerven T, Jones P T, Binnemans K (2017). REE recovery from end-of-life $\mathrm{NdFeB}$ permanent magnet scrap: A critical review. Journal of Sustainable Metallurgy, 3(1): 122149

Yao Y C, Taqieddin A, Alibakhshi M A, Wanunu M, Aluru N R, Noy A (2019). Strong electroosmotic coupling dominates ion conductance of $1.5 \mathrm{~nm}$ diameter carbon nanotube porins. ACS Nano, 13(11): 12851-12859

Zeidel M L, Ambudkar S V, Smith B L, Agre P (1992). Reconstitution of functional water channels in liposomes containing purified red cell CHIP28 protein. Biochemistry, 31(33): 7436-7440

Zhang X, Fu W, Palivan C G, Meier W (2013). Natural channel protein inserts and functions in a completely artificial, solid-supported bilayer membrane. Scientific Reports, 3(1): 2196

Zhang X, Tanner P, Graff A, Palivan C G, Meier W (2012). Mimicking the cell membrane with block copolymer membranes. Journal of Polymer Science. Part A, Polymer Chemistry, 50(12): 2293-2318

Zhao H, Ong W Q, Fang X, Zhou F, Hii M N, Li S F Y, Su H, Zeng H (2012a). Synthesis, structural investigation and computational modelling of water-binding aquafoldamers. Organic \& Biomolecular Chemistry, 10(6): 1172-1180

Zhao H, Sheng S, Hong Y, Zeng H (2014a). Proton gradient-induced water transport mediated by water wires inside narrow aquapores of aquafoldamer molecules. Journal of the American Chemical Society, 136(40): 14270-14276

Zhao J, Zhao X, Jiang Z, Li Z, Fan X, Zhu J, Wu H, Su Y, Yang D, Pan F, Shi J (2014b). Biomimetic and bioinspired membranes: Preparation and application. Progress in Polymer Science, 39(9): 1668-1720

Zhao Y, Qiu C, Li X, Vararattanavech A, Shen W, Torres J, HélixNielsen C, Wang R, Hu X, Fane A G, Tang C Y (2012b). Synthesis of robust and high-performance aquaporin-based biomimetic membranes by interfacial polymerization-membrane preparation and RO performance characterization. Journal of Membrane Science, 423424: 422-428

Zhong P S, Chung T S, Jeyaseelan K, Armugam A (2012). Aquaporinembedded biomimetic membranes for nanofiltration. Journal of Membrane Science, 407-408: 27-33

Zhou X, Liu G, Yamato K, Shen Y, Cheng R, Wei X, Bai W, Gao Y, Li H, Liu Y, Liu F, Czajkowsky D M, Wang J, Dabney M J, Cai Z, Hu J, Bright F V, He L, Zeng X C, Shao Z, Gong B (2012). Selfassembling subnanometer pores with unusual mass-transport properties. Nature Communications, 3(1): 949

Zhu F, Tajkhorshid E, Schulten K (2004). Collective diffusion model for water permeation through microscopic channels. Physical Review Letters, 93(22): 224501 